\title{
Anatomical Correlates of Functional Plasticity in Mouse Visual Cortex
}

\author{
Antonella Antonini, ${ }^{1}$ Michela Fagiolini,, ${ }^{1,2}$ and Michael P. Stryker ${ }^{1}$ \\ 1W. M. Keck Foundation Center for Integrative Neuroscience, Department of Physiology, University of California, San \\ Francisco, California 94143-0444, and 2Laboratory for Neuronal Circuit Development, Brain Science Institute, RIKEN, \\ Saitama 351-01, Japan
}

Much of what is known about activity-dependent plasticity comes from studies of the primary visual cortex and its inputs in higher mammals, but the molecular bases remain largely unknown. Similar functional plasticity takes place during a critical period in the visual cortex of the mouse, an animal in which genetic experiments can readily be performed to investigate the underlying molecular and cellular events. The experiments of this paper were directed toward understanding whether anatomical changes accompany functional plasticity in the developing visual cortex of the mouse, as they do in higher mammals. In normal mice, transneuronal label after an eye injection clearly delineated the monocular and binocular zones of area 17. Intrinsic signal optical imaging also showed monocular and binocular zones of area 17 but revealed no finer organization of ocular dominance or orientation selectivity. In normal animals, single geniculocortical afferents serving the contralateral eye showed great heterogeneity and no clustering consistent with the presence of ocular dominance patches. Growth and elaboration of terminal arbor continues beyond postnatal day 40

The organization, development, and plasticity of the primary visual cortex are of interest for its role in understanding visual perception, but the cortex is of even more widespread interest as a model system for studying how patterns of neural activity operate to organize connections in the developing brain. Much of our understanding of how neural connections are modified by sensory experience stems from the remarkable rearrangements of physiological responses and anatomical connections produced by blurring or occluding vision in one eye during a sensitive period in early life (Hubel et al., 1977; LeVay et al., 1980).

In animals with binocular vision, there is a region of visual cortex within which single neurons respond to stimulation of both eyes and a more peripheral representation that responds to visual stimulation only through the contralateral eye. In many species, inputs representing the two eyes that arise from the lateral geniculate nucleus (LGN) and project to the binocular zone of visual cortex are arranged in alternating patches in layer IV, from which they preferentially activate the radial columns of cortical

\footnotetext{
Received Sept. 29, 1998; revised Jan. 21, 1999; accepted Feb 24, 1999.

This work was supported by National Institutes of Health Grant NS16033 to M.P.S. We thank Sheri Harris for technical support, Jessica Hanover for participating in the data collection for Fig. 1, and Dr. Takao K. Hensch for critical discussions and support.

Correspondence should be addressed to Prof. Michael P. Stryker, Department of Physiology, Room S-762, Box 0444, 513 Parnassus Avenue, University of California, San Francisco, California 94143-0444.

Copyright (C) 1999 Society for Neuroscience $\quad 0270-6474 / 99 / 194388-\bullet \$ 05.00 / 0$
}

(P40), after the peak of the critical period. After prolonged monocular deprivation (MD) from P20 to P60, transneuronal labeling showed that the projection serving the ipsilateral eye was severely affected, whereas the effect on the contralateral eye's pathway was inconsistent. Optical imaging also showed profound effects of deprivation, particularly in the ipsilateral pathway, and microelectrode studies confirmed continued functional plasticity past P40. Reconstruction of single afferents showed that MD from P20 to P40 promoted the growth of the open eye's geniculocortical connections without causing the closed eye's contralateral projection to shrink, whereas MD from P20 to P60 caused an arrest of growth of deprived arbors. Our findings reveal numerous similarities between mouse and higher mammals in development and plasticity, along with some differences. We discuss the factors that may be responsible for these differences.

Key words: area V1; optical imaging; monocular deprivation; critical period; area 17; development; visual cortex; transneuronal labeling; axonal reconstruction cells above and below, an arrangement referred to as ocular dominance columns (Hubel and Wiesel, 1965; Shatz and Stryker, 1978; LeVay et al., 1980). If, during the critical period in early life, one eye is allowed to see normally, whereas the vision of the other eye is occluded [monocular deprivation (MD)], most of the cortical cells, even those in the deprived eye's columns, lose their ability to respond to the deprived eye (cat: Wiesel and Hubel, 1963; Hubel and Wiesel, 1970; Hubel et al., 1977; Shatz and Stryker, 1978; monkey: LeVay et al., 1980; rat: Fagiolini et al., 1994). This physiological loss of response is followed in $<1$ week by a dramatic retraction of the branches of deprived geniculocortical arbors and is followed much later by a compensatory expansion of the arbors of the open eye (LeVay et al., 1980; Antonini and Stryker, 1996). This process depends on a competitive interaction between patterns of neural activity in the two eyes' pathways in the cortex, and it does not take place when the vision of both eyes is occluded or when activity in the cortex is blocked (Wiesel and Hubel, 1963, 1965a; Guillery and Stelzner, 1970; Guillery, 1973; Stryker and Harris, 1986; Antonini and Stryker, 1998). The effects of deprivation can be reversed to a limited extent during the critical period by reversing the visual deprivation, but they later become irreversible (Wiesel and Hubel, 1965b; Movshon, 1976; van Sluyters, 1978; Blakemore et al., 1981; Antonini et al., 1998). Finally, the outcome of deprivation depends on cortical activity, because MD causes the opposite effect on ocular dominance if the cortical cells are pharmacologically in- 
Table 1. Arbors reconstructed in normal and MD animals

Normal animals

\begin{tabular}{|c|c|c|c|c|c|c|c|}
\hline & \\
\hline Exp. & $\begin{array}{l}\text { Age at per- } \\
\text { fusion }\end{array}$ & Arbors & Exp. & $\begin{array}{l}\text { Days of } \\
\text { deprivation }\end{array}$ & $\begin{array}{l}\text { Age at per- } \\
\text { fusion }\end{array}$ & Deprived arbors & Nondeprived arbors \\
\hline mo1 & P35 & mo1a, mo1b & D2 & 17 & P37 & & $\mathrm{d} 2 \mathrm{nda}, \mathrm{d} 2 \mathrm{ndb}, \mathrm{d} 2 \mathrm{ndc}, \mathrm{d} 2 \mathrm{ndc}$ \\
\hline \multirow[t]{2}{*}{ mo2 } & P35 & $\operatorname{mo} 2 \mathrm{a}, \operatorname{mo} 2 \mathrm{~b}, \operatorname{mo} 2 \mathrm{c}, \operatorname{mo} 2 \mathrm{~d}$ & D7 & 19 & P39 & & d7nda, d7ndb \\
\hline & & $\mathrm{mo} 2 \mathrm{e}, \mathrm{mo} 2 \mathrm{f}, \mathrm{mo} 2 \mathrm{~g}, \mathrm{mo} 2 \mathrm{~h}$ & D3 & 18 & P39 & $\mathrm{d} 3 \mathrm{a}, \mathrm{d} 3 \mathrm{~b}$ & \\
\hline mo3 & $\mathrm{P} 40$ & mo3a, mo3b, mo3c, mo3d & D5 & 19 & P39 & $\mathrm{d} 5 \mathrm{a}, \mathrm{d} 5 \mathrm{~b}$ & \\
\hline \multirow[t]{5}{*}{ mo4 } & P60 & mo4a, mo4b, mo4c, mo4d, mo4e & D8 & 19 & P39 & $\mathrm{d} 8 \mathrm{a}, \mathrm{d} 8 \mathrm{~b}$ & \\
\hline & & & D1 & 36 & P56 & & d1nda, d1ndb, d1ndc, d1ndd \\
\hline & & & D6 & 39 & P59 & & d6nda, d6ndb \\
\hline & & & D4 & 39 & P60 & $\begin{array}{l}\text { d4da, d4db } \\
\text { d4ndc, d4ndd } \\
\text { d4nde }\end{array}$ & \\
\hline & & & D9 & 44 & P64 & d9da, d9db & \\
\hline
\end{tabular}

hibited (Reiter and Stryker, 1988; Hata and Stryker, 1994). In the cat, all of these plasticity phenomena have been demonstrated physiologically (Wiesel and Hubel, 1965a,b) and anatomically, both at the level of the entire geniculocortical projection (studied by transneuronal labeling) and at the level of single geniculocortical afferent arbors (Shatz and Stryker, 1978; Hata and Stryker, 1994; Antonini and Stryker, 1996, 1998).

Understanding the mechanisms responsible for activitydependent plasticity is a major goal of neuroscience because these same mechanisms are thought to guide the development of precise connections in many regions of the normal brain (Goodman and Shatz, 1993). The higher mammals in which these phenomena have been studied are, unfortunately, unsuitable for genetic manipulation. For this reason, the mouse has attracted increasing interest as a species for study of the molecular and cellular machinery responsible for plasticity (Fagiolini et al., 1998; Hensch et al., 1998a,b).

Recently, we extended the earlier findings of Dräger (1975, 1978) by demonstrating a rapid, competitive physiological plasticity during a critical period in the developing mouse visual cortex similar to the plasticity in higher mammals (Gordon and Stryker, 1996). Even more recent studies in transgenic animals have shown the promise of this system, which would be greater if it were understood in more depth and if anatomical information were available for comparison with data from higher mammals (Fagiolini et al., 1998; Hensch et al., 1998a).

In this paper we examine the arrangement of geniculocortical inputs labeled transneuronally and the shapes of individual afferent arbors serving the contralateral eye, both during normal development and after plasticity induced by monocular deprivation. We relate the anatomical findings to visual cortical plasticity assayed with two different physiological measures: intrinsic signal optical imaging and extracellular microelectrode recording. Our findings reveal numerous similarities between the mouse and higher mammals in development and plasticity, as well as some differences. We discuss the factors that may be responsible for these differences.

Some of these results have been presented in abstract (Fagiolini et al., 1997).

\section{MATERIALS AND METHODS}

All mice studied were C57Bl/6 (BNT) and were housed in the University of California (San Francisco, CA). Experimental procedures were approved by the institutional animal care and use committee.

\section{Tracer injections into the LGN, anterograde labeling, and reconstruction of geniculocortical arbors}

Tracer injections. The aim of the experiment was to reconstruct single geniculocortical axons serving the contralateral eye and innervating the binocular portion of the visual cortex in normal and monocularly deprived mice. To this end, anterograde tracers were injected at LGN sites identified electrophysiologically.

Normal mice were studied at postnatal days 35-40 (P35-P40) or at P60. Monocular deprivations lasted for 20 or $40 \mathrm{~d}$ beginning at P17-P19 (Table 1). Animals were prepared for electrophysiological recordings following the procedure described by Gordon and Stryker (1996), with slight modifications. Briefly, mice were anesthetized with an intraperitoneal injection of $50 \mathrm{mg} / \mathrm{kg}$ pentothal (Nembutal; Abbott, North Chicago, IL). To hasten recovery at the end of recordings, only the single dose of pentothal was administered, after which anesthesia was maintained with $1-1.5 \%$ halothane in a $1: 1$ mixture of $\mathrm{N}_{2} \mathrm{O} / \mathrm{O}_{2}$. Dexamethasone $(0.05 \mathrm{mg}$; Steris Laboratories, Phoenix, AZ) and atropine ( $0.3 \mathrm{mg}$, Atroject; Burns, Rockville Centre, NY) were administered subcutaneously. Temperature was monitored by a rectal thermometer and maintained at $37^{\circ} \mathrm{C}$ by means of a heating pad. The electrocardiogram was also continuously recorded through leads attached to the right forelimb and left hindlimb. The animals' eyes were protected with silicone oil.

A small flap of bone $(3 \times 3 \mathrm{~mm})$ was removed over the rostral half of the hemipheres, and the dura was left intact. To protect the visual cortex from damage, care was taken not to expose fully the caudal portion of the brain. A glass pipette filled with the tracer was positioned under visual guidance onto the surface of the brain at the stereotaxic coordinates anteroposterior 2.0-2.3 posterior to bregma and mediolateral 3-3.3 from the midline (Franklin and Paxinos, 1997; the boundaries of area 17 in our mice did not correspond to those shown in this atlas, but the LGN coordinates did). The pipette was slowly advanced through the brain while light stimuli were projected onto a screen positioned $30 \mathrm{~cm}$ in front of the animal and at an angle of $58^{\circ}$ from the midline. When single- or multi-unit activity was clearly driven by visual stimulation, we proceeded to map the location of the receptive field on the screen. In the mouse, the region of the visual space seen by both eyes comprises the central $30-40^{\circ}$ of the upper visual field (Dräger, 1975; Gordon and Stryker, 1996). We chose to inject the anterograde tracer into regions of the LGN containing the representation of the central $20-30^{\circ}$ of the upper visual field, that is, within the anterior half of the nucleus. To label selectively geniculocortical afferents serving the contralateral eye, we always verified that the site chosen for tracer injection was driven only through the contralateral eye. The injection was performed $50-100 \mu \mathrm{m}$ ventral to the first visual response obtained while lowering the recording pipette. At this location the injection site was usually limited to the dorsal surface of the LGN, which receives projections only from the contralateral eye. Biocytin (Sigma, St. Louis, MO; $2.5 \%$ in saline) was iontophoretically injected into the LGN using a high-voltage current source device (Midgard Electronics; Stoelting, Wood Dale, IL) with a positive current (4 $\mu \mathrm{A}$, 5-15 min). In one animal (mo3; Table 1) the Phaseolus lectin Pha-L (2.5\% solution in $0.1 \mathrm{~m}$ PBS, $\mathrm{pH} 8$; Vector Laboratories, Burlingame, CA) was iontophoretically injected $(8 \mu \mathrm{A}, 4 \mathrm{~min})$. Only a single injection 
in each LGN was made. At the end of the experiment, the bone flap was repositioned, and the overlying skin was sutured.

In all MD animals and in animal mo4 (Table 1) one eye (the deprived eye in the MD animals) was injected with the rhodamine fluorescent dextran Fluoro Ruby (Molecular Probes, Eugene, OR) to verify the position of the injection site relative to the LGN laminae. In all cases in which biocytin was used, injection sites were well defined and were limited to the dorsal portion of the LGN, far from the zone receiving ipsilateral input. In the Pha-L case (mo3), the injection site appeared more diffuse, and we cannot exclude the possibility that ipsilateral eye's pathways were involved. However, the bulk of the Pha-L injection was located in the dorsal portion of the LGN, and we therefore included the four geniculocortical arbors obtained from this animal in the normal analysis.

Histological processing. After a survival time of 2-3 d, the animals were deeply anesthetized with pentothal and perfused through the heart with $0.1 \mathrm{M}$ phosphate buffer followed by $4 \%$ paraformaldehyde in $0.1 \mathrm{M}$ phosphate buffer. The brains were cut on a vibratome at $40 \mu \mathrm{m}$ in the coronal plane, and sections from the posterior portion of the hemisphere containing the visual cortex were reacted for biocytin histochemistry or for PHA-L immunohistochemistry.

In experiments in which biocytin was injected, sections through the visual cortex were first incubated in $50 \%$ ethanol with $0.1 \%$ hydrogen peroxide $(15 \mathrm{~min})$, washed in $0.05 \mathrm{M}$ Trizma-saline buffer, $\mathrm{pH} 7.4$, with $0.3 \%$ Triton X-100 (four times for $10 \mathrm{~min}$ each), and finally incubated overnight with avidin-biotin-HRP complex (Vector) in Trizma buffer. The following day, sections were reacted with $0.05 \%$ diaminobenzidine hydrochloride (Sigma), $0.7 \%$ nitroammonium sulfate (Sigma), and $0.03 \%$ of hydrogen peroxide in $0.05 \mathrm{~m}$ Trizma buffer, $\mathrm{pH}$ 7.4. Immunohistochemical procedures for the Pha-L case are described in detail by Antonini and Stryker (1996).

Sections through the LGN were first photographed in fluorescence microscopy to identify areas receiving the contralateral or ipsilateral retinal fibers (see Fig. $5 A$ ). The sections were then placed for $20 \mathrm{~min}$ in PBS containing $0.3 \%$ Triton X-100, reacted with avidin-conjugated fluorescein $(0.0025 \%$ in PBS) for 1-2 hr, and finally washed for $20-30 \mathrm{~min}$ in PBS. Wet sections containing the injection site were photographed or scanned with a confocal microscope. This procedure allowed us to evaluate the location of the injection site relative to the ipsilateral and contralateral retinogeniculate projections by comparing the photographs of the same section before and after the avidin-fluorescein reaction (see Fig. $5 B$ ). Only those injection sites entirely confined to the dorsal portion of the LGN, with no involvement of the area containing projections from the ipsilateral eye, were considered in this study.

Both the photographic slides and the confocal images were scanned and processed with the aid of Photoshop image analysis software (Adobe Systems, Mountain View, CA). This photographic procedure was necessary to identify the lamination of the LGN in relation to injection sites, because the rhodamine fluorescence of the retinal projections became faint after the avidin-fluorescein reaction.

Axonal reconstructions. Geniculocortical arbors were reconstructed in three dimensions at $1000 \times$ from serial sections with the aid of a computer graphic system described previously (Neurotrace; InterAction, Boston, MA; Passera et al., 1988; Antonini and Stryker, 1996).

Two measurements were used to quantify the features of the terminal arborization of the reconstructed afferents (Antonini and Stryker, 1996): (1) Total linear length of the arborization. This value was obtained by the addition of the three-dimensional lengths of all the branches constituting the terminal field of an arbor. Only the portions of the arbor located in layer IV and in the supragranular layers were considered for this measure. Terminal branches $<5 \mu \mathrm{m}$ were excluded from the analysis, because it was unclear whether the shortest processes were branches or boutons. Such an exclusion had no significant effect on the measurement of total length, but it was necessary for the counts of branch points to be consistent from observer to observer. (2) Number of branch points of the terminal arborization. This value was obtained from the arborization in layer IV and supragranular layers. Again, branch points giving rise to terminals $<5 \mu \mathrm{m}$ in length were excluded.

For both measures, evaluation of the differences among groups was obtained by comparing groups two at a time, using the Mann-Whitney $U$ test for nonparametric statistical analyses.

\section{Transneuronal labeling of geniculocortical projections}

Wheat germ agglutinin conjugated to horseradish peroxidase (WGAHRP, $1.5 \%, 2-3 \mu \mathrm{l}$; Sigma) in saline was injected into one eye. After a survival time of 3-5 d, the animals were perfused with $0.1 \mathrm{M}$ phosphate buffer followed by $2 \%$ glutaraldehyde in $0.1 \mathrm{M}$ phosphate buffer. The cerebral hemispheres were flattened, sandwiched between two glass slides, and cryoprotected overnight in $30 \%$ sucrose in $0.1 \mathrm{M}$ phosphate buffer containing $2 \%$ glutaraldehyde. The next day the flattened hemispheres were cut tangentially at $40 \mu \mathrm{m}$ and reacted by the tetramethylbenzydine method (Mesulam, 1978), and photographed in dark field as soon as possible. Negatives were scanned, and Figures $2-5$ were assembled with the aid of Photoshop.

\section{Monocular deprivation}

Animals were anesthetized with $2.0-2.5 \%$ halothane in a 1:1 mixture of $\mathrm{N}_{2} \mathrm{O} / \mathrm{O}_{2}$. The area around the eye was wiped with $70 \%$ ethanol, and the superior and inferior eyelids were trimmed along the margins and then sutured with 7-0 sterile surgical silk (Ethicon, Somerville, NJ). The animals were checked every day for the first 4-5 d to make sure that the eyes remained closed. Subsequently, when the scar tissue had permanently sealed the eyelids, animals were checked every other day. The eye was reopened for several hours for the tracer injection into the LGN and the intraocular injection of rhodamine dextran and subsequently resutured.

Table 1 lists for each animal the duration of the MD and the age at perfusion.

\section{Single-unit recordings}

Five normal mice between P45 and P60, three mice monocularly deprived from $\mathrm{P} 20$ to $\mathrm{P} 40$, three mice deprived from P20 to P60, and three mice deprived from P40 to P60 were used for single-unit electrophysiological recordings. The animals were prepared for electrophysiological recordings in a manner similar to that described by Gordon and Stryker (1996). Briefly, the animals were anesthetized by intraperitoneal injection of $50 \mathrm{mg} / \mathrm{kg}$ pentothal and maintained on pentothal as needed. A tracheotomy was performed, and the animal breathed spontaneously a mixture of oxygen and room air through a capillary tube inserted into the trachea. The head was placed in a stereotaxic holder, and temperature and electrocardiogram were continuously monitored as described above. The eyelids were trimmed to expose the entire pupil, and the corneas were protected with silicone oil. A bone flap was removed above the posterior half of the brain; the dura was left intact. Resin-coated tungsten electrodes were used to record single units. The electrode was positioned, under visual guidance, in the binocular zone of the visual cortex (Dräger, 1975; Wagor et al., 1980; Gordon and Stryker, 1996). The cortex was covered with $3 \%$ agarose in saline. Visual stimuli consisting of oriented light bars moved by hand in different directions were presented on a screen positioned $30 \mathrm{~cm}$ from the animal's eye at an angle of $58^{\circ}$ from the midline. Neurons were classified into the seven traditional ocular dominance groups according to Hubel and Wiesel (1962). The Contralateral Bias Index (CBI) was used to summarize the ocular preference. This index is a weighted average toward one or the other eye and is calculated by the formula: $\mathrm{CBI}=[(n 1-n 7)+2 / 3(n 2-n 6)+1 / 3(n 3-n 5)+$ $N] / 2 N$, where $N$ is the total number of cells and $n x$ is the number of cells in an ocular dominance group $x$. CBI values of 1.00 and 0.00 represent complete dominance by the contralateral or the ipsilateral eye, respectively.

\section{Intrinsic signal optical imaging}

Four normal mice between P35 and P40 and four P40 mice monocularly deprived for $20 \mathrm{~d}$ were used in optical imaging experiments. The animals were prepared as for single-unit recordings, with the addition of computer-controlled shutters in front of each eye. The dura was left intact, and the exposed brain was covered with $3 \%$ agarose and sealed with a clear glass coverslip to ensure a flat surface. Details of the optical imaging apparatus and protocols have been published previously (Crair et al., 1997). The cortical surface was illuminated with a green light, and the camera (Princeton Instruments, Trenton, NJ) was initially focused on the pial surface to obtain a clear image of the blood vessels over a $3.2 \times$ $2.4 \mathrm{~mm}$ field. For acquiring intrinsic cortical signals, the cortical surface was illuminated with $610 \mathrm{~nm}$ light, and the camera was focused at $150-400 \mu \mathrm{m}$ below the pial surface. Visual stimuli were square wave gratings $\left(0.05\right.$ or $\left.0.1 \mathrm{c} /{ }^{\circ}\right)$ moving for $6 \mathrm{sec}$ alternately in the two directions at four different orientations $\left(0,45,90\right.$, and $\left.135^{\circ}\right)$ and viewed monocularly and were separated by $6 \mathrm{sec}$ interstimulus intervals. Stimuli appeared stationary on the screen at the beginning of each interstimulus interval, and optical signals from the cortex were collected in $10600 \mathrm{msec}$ image frames beginning at the onset of stimulus motion. The different 
stimulus orientations and eyes stimulated were interleaved in random order on a monitor placed $40 \mathrm{~cm}$ in front of the animal. For each run, the eight stimuli (four orientations $\times$ two eyes) plus two blank-screen stimuli isoluminant with the gratings were presented 16 times in a random order.

For each stimulus orientation and for each eye, the average raw image from all orientation was divided by the average blank-screen stimulus to produce the functional image. Brain areas responding to the stimulus increased absorption and thereby became darker on the functional maps. All functional maps from each hemisphere were thresholded identically, allowing quantitative comparison among them. For comparing the responses to the two eyes in a single hemisphere, ocular dominance ratio maps were computed by dividing the summed raw images from all stimuli delivered to one eye by a similar sum for the other eye.

\section{RESULTS}

\section{The visual cortex in the mouse: localization and normal histology}

Electrophysiological recordings have revealed a single continuous representation of the monocular and binocular segments of the visual field within the primary visual cortex of the mouse (Dräger; 1975, 1978; Wagor et al., 1980; Gordon and Stryker, 1996). A number of histological features of the cortex have been shown in many species, including rodents, to distinguish area 17 from the surrounding cortical areas. We wished to determine whether these various histological features were in register with one another in the visual cortex of the mouse and whether they corresponded to the region of cortex delineated by its topographic map of the visual field.

Figure 1 shows two hemispheres from a P38 mouse in which serial sections have been processed with Nissl, acetylcholinesterase, and myelin stains and placed in register. In Nissl-stained coronal sections, the primary visual area can be distinguished by the presence of densely packed granule cells in layer IV (Valverde, 1968; Caviness, 1975). The monocular portion can also be differentiated from the binocular portion, although not as easily as in the rat (Zilles et al., 1984), by the greater regularity in the organization of layer IV granule cells. These features are evident in Figure 1, $A$ and $B$. Acetylcholinesterase staining (Hedreen et al., 1985) can also be used in many species to delineate area 17 from surrounding areas by its characteristically high activity in layers III and IV. Figure $1, C$ and $D$, shows that such heightened activity distinguishes area 17 in the mouse and corresponds closely to the borders of area 17 evident in the Nissl stain. In addition, the binocular segment of area 17 is stained somewhat more intensely than the monocular segment, and the stain extends more superficially. Myelin staining (Gallyas, 1979) also gives a clear indication of the location of area 17 in several species, including rodents (Zilles et al., 1984; Olavarria and Montero, 1989). Figure 1, $E$ and $F$, shows agreement in the mouse between the borders of area 17 delineated by the myelin pattern and those defined by the other stains. Specifically, the medial, monocular portion of area 17 is characterized by myelinated radial fibers extending up to layer II/III, crossed by two longitudinal plexi in layers IV and V. A few fibers also run longitudinally in layer I. Laterally, in the binocular segment, the myelin is denser, with more profuse staining between the two fiber plexi.

Microelectrode recordings confirmed the histological identification of area 17. Electrode penetrations revealed a topographic map as reported previously (Dräger, 1975, 1978; Wagor et al., 1980; Gordon and Stryker, 1996). The lateral border of area 17, at the point of reversal of the progression of receptive field azimuths, was marked with small electrolytic lesions ( $5 \mu \mathrm{A}$ for $5 \mathrm{sec})$ seen in Figure $1, B, D$, and $F$. This physiologically defined border coincided with the border as defined histologically. The medio- lateral extent of the binocular segment also agreed with the size of the binocular segment mapped electrophysiologically.

\section{Transneuronal labeling of thalamocortical input in normal animals}

Area 17 is also defined by its receipt of a direct projection from the LGN. We studied the pattern of termination of the geniculocortical pathway using the transneuronal transport of WGA-HRP injected into one eye (Itaya and van Hoesen, 1982) in four P40 and 4 P60 normal mice. In each age group, the brains of two animals were flattened and cut tangentially to the pial surface, whereas the other two brains were cut in the coronal plane. Figure 2 shows transneuronal labeling of geniculocortical afferents in a series of coronal sections through the hemispheres contralateral and ipsilateral to the injected eye in a P60 mouse. Clear labeling was observed in both the monocular and binocular portions of area 17 in the contralateral hemisphere and in the binocular segment of the ipsilateral hemisphere (Dräger, 1974). In both hemispheres, labeling in the binocular portion appeared nearly homogeneous, with no clear sign of the large ocular dominance patches typical of layer IV of area 17 in higher mammals (Dräger, 1974; Shatz and Stryker, 1978; LeVay et al., 1980).

In the caudal pole of the forebrain, area 17 curved around the ventral surface of the hemisphere, whereas in successive posteroanterior sections, it covered progressively more lateral portions of the hemisphere. Furthermore, no projection from the ipsilateral eye was found in the rostral-most sections where the lower, monocular portion of the visual field is represented.

It has been reported that in rodents the LGN projections terminate exclusively in area 17 and do not invade the surrounding areas 18b and 18a (Ribak and Peters, 1975; Caviness and Frost, 1980; Simmons et al., 1982). Our experiments confirm that the bulk of the transneuronal labeling is confined to area 17 , identified cytoarchitectonically after counterstaining for Nissl in preparations such as that of Figure 3. The medial border of the transneuronal label is typically very sharp, suggesting that no geniculocortical projections reach area $18 \mathrm{~b}$. However, a very light transneuronal labeling, clearly above background, was present lateral to area 17 in area 18a (Fig. 3, bottom), indicating that a small number of LGN projections reach this area.

In a few coronal sections from some experiments (Fig. 3, bottom), the binocular zone in the hemisphere contralateral to the injected eye appeared paler than the monocular zone. This result is in agreement with the report by Dräger (1974) in her transneuronal study using tritiated proline, where she suggested that this pattern of labeling indicates that the full innervation of the binocular zone can only be exhibited by labeling the projections from both eyes.

The whole extent of the primary visual area, labeled transneuronally after an intraocular injection of WGA-HRP, can be appreciated in tangential sections through the flattened hemisphere. Figure 4 shows the hemispheres contralateral and ipsilateral to the injected eye in a P40 mouse. On the contralateral side, the labeled area had a triangular shape with a sharp border on the medial side. In the hemisphere ipsilateral to the injected eye, transneuronal label was always present and restricted to a patch in the lateral portion of the hemisphere.

\section{Transneuronal labeling of the geniculocortical projection in monocularly deprived animals}

In many other species, prolonged monocular deprivation alters the distribution and intensity of transneuronally labeled genicu- 

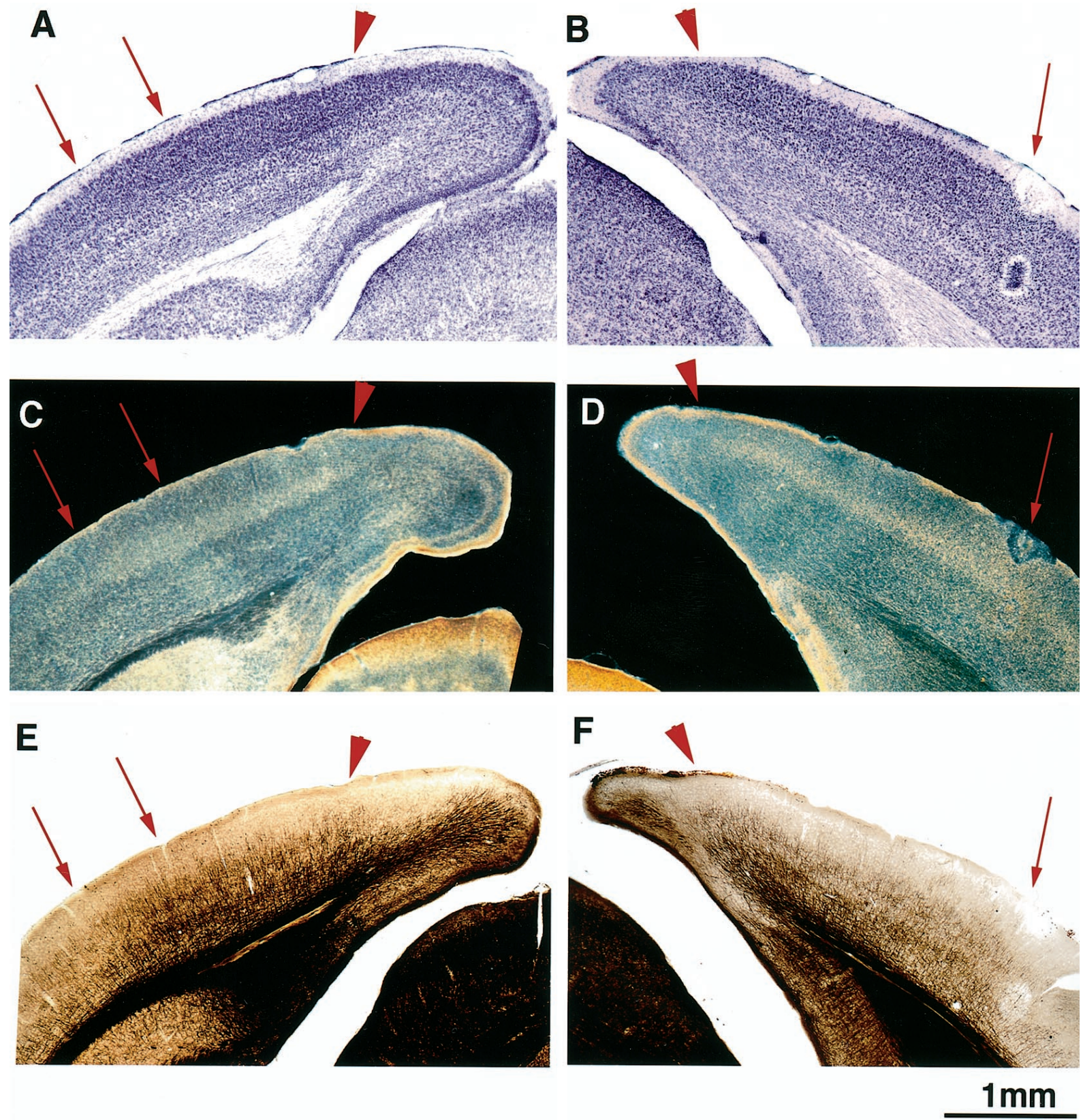

Figure 1. A, C, E, Consecutive $50 \mu \mathrm{m}$ sections through the posterior pole of the cerebral hemisphere. $B, D, F$, Consecutive sections from the opposite hemisphere of the same mouse in which an electrolytic lesion (red arrow) was made after electrophysiological recordings to mark the border between areas 17 and 18a. Area 17 is recognizable in Nissl staining by the presence of small granule cells in layer IV (A, black arrow). High acetylcholinesterase activity in layer IV is also a marker for the primary visual cortex $(C, D$, light staining), as is the pattern of myelin staining $(E, F)$. The medial border of the visual cortex is clearly identified in all three stains (arrowhead). Furthermore, there is a good match between the 17-18a border recognized in the three stains and that identified electrophysiologically $(B, D, F)$. The pair of arrows on the left point to the binocular portion of the visual cortex clearly recognizable in all three preparations.

locortical projections in layer IV of the visual cortex (Shatz and Stryker, 1978; LeVay et al., 1980). Here we have adopted the same technique in the mouse to investigate whether the pattern of transneuronal label in the ipsilateral and contralateral hemispheres was modified in MD animals. Eight mice were monocularly deprived at P20 and perfused at P60. Three to $5 \mathrm{~d}$ before perfusion, each animal received an intraocular injection of WGA-HRP into the deprived or the nondeprived eye (see Materials and Methods). The hemispheres were flattened and cut in the tangential plane. Geniculocortical terminations in the hemi- sphere ipsilateral to the injected eye were consistently and strongly affected by MD. In two of the four animals in which WGA-HRP was injected into the deprived eye, the transneuronal labeling in the ipsilateral hemisphere was clearly evident but was pale and spatially restricted compared with normal animals (Fig. 5, ltd8, ltd5). Transneuronal labeling in the other two animals (Fig. 5, ltd4, ltd1) was so faint it was not clearly detectable, presumably because the sensitivity of the technique does not allow detection of a very modest signal. By contrast, in all the cases in which the nondeprived eye was injected, the ipsilateral 


\section{contralateral}

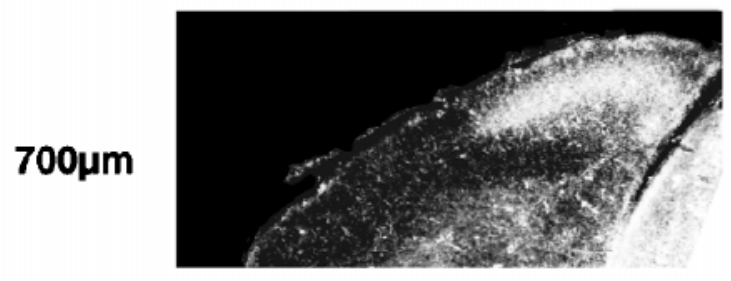

$1100 \mu \mathrm{m}$

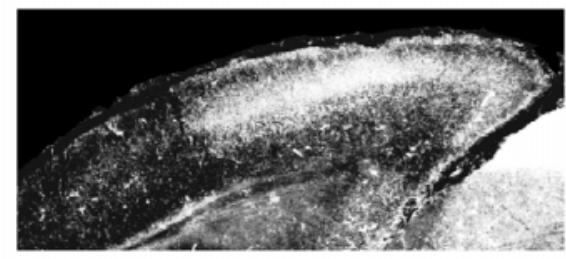

1500um

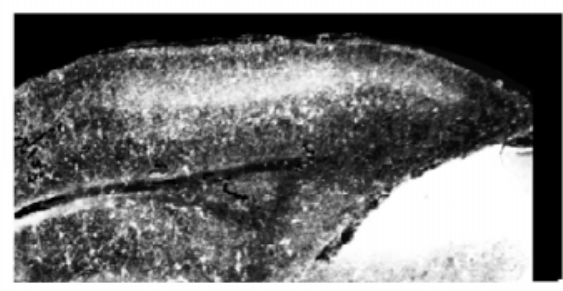

1700um

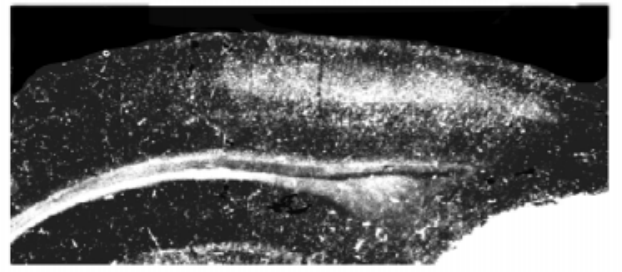

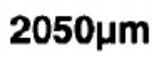

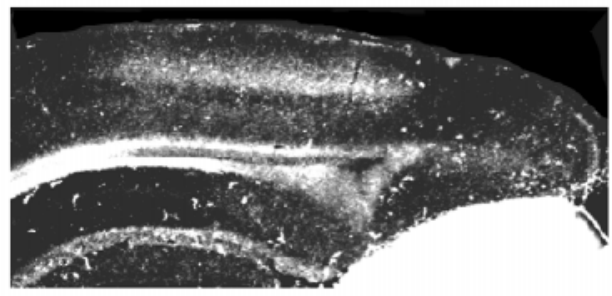

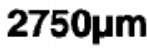

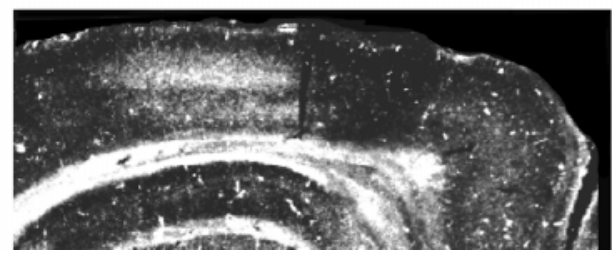

ipsilateral
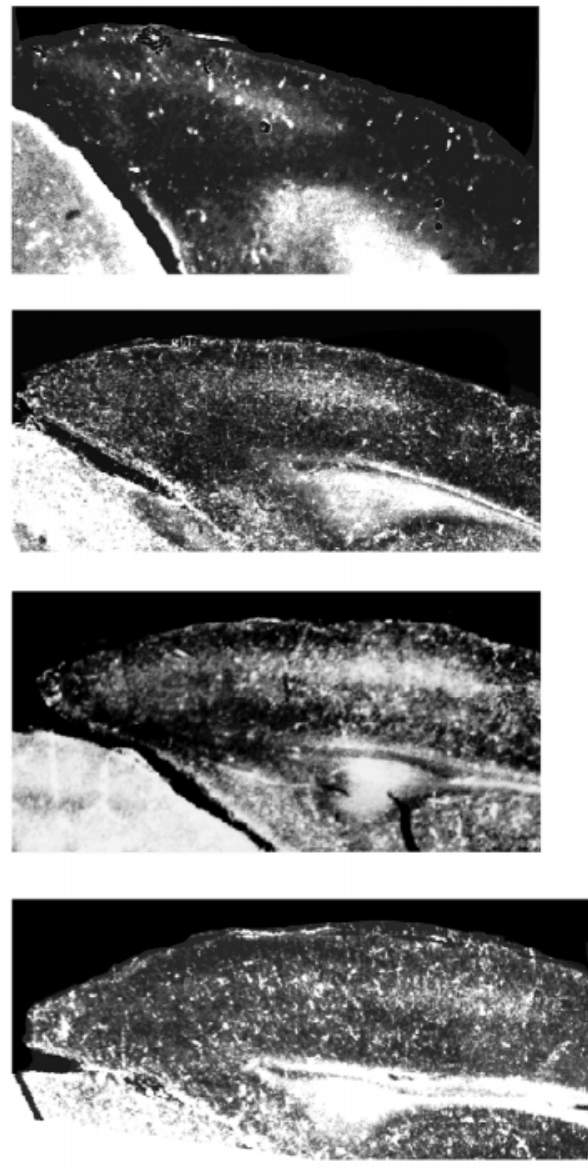

$1 \mathrm{~mm}$

Figure 2. Extent of the mouse visual cortex in the coronal plane, as demonstrated by the transneuronal transport of WGA-HRP injected into one eye. The series of coronal sections through the hemispheres contralateral and ipsilateral to the eye injected with WGA-HRP are presented from posterior to anterior; the distance from the posterior edge of the hemispheres is indicated on the left. The images have been obtained by scanning the photographic negatives of the histological sections. The light intensity and contrast of the images of the ipsilateral hemisphere (right column) have been enhanced to better visualize the ipsilateral projections. hemisphere contained a strong transneuronal labeling (Fig. 5, ltd2, ltd3, ltd6, ltd7) that was at least as strong as in normal animals. In the hemisphere contralateral to the injected eye, transneuronal labeling was generally strong after injection of either the deprived or nondeprived eye. In all cases, with the exception of ltd1 (Fig. 5), careful examination of all the sections through each flattened hemisphere failed to reveal a consistent difference in the strength of the trasneuronal labeling between the medial and lateral portions of the labeled area, corresponding to the monocular and binocular zone of area 17. Case ltd 1 differed in that the lateral third of the transneuronally labeled area, which we presume is the binocular zone of area 17, was much paler than the medial, monocular zone. This feature has been previously described in normal animals (see text above; Fig. $3 B$; Dräger, 1974). However, such a steep gradient between the monocular and binocular segments was never observed in our normal animals and is consistent with a loss of geniculocortical terminals serving the contralateral eye after MD.

In a group of eight P40 animals monocularly deprived for $20 \mathrm{~d}$, the contralateral projections from either the deprived or nondeprived eyes appeared within the normal range. The labeling of the ipsilateral projection from the deprived eye was not consistently less intense than normal, except in one of the four cases, in which it was very pale. 
Figure 3. Aligned, consecutive $40 \mu \mathrm{m}$ sections from a P43 animal in which one eye was injected with WGAHRP. Top, Nissl stain. Area 17 is recognizable cytoarchitectonically by the presence of small and closely packed cells in layer IV, more evident in the monocular $(m)$ than in the binocular region, enclosed by the two gray arrows (b). The transneuronal WGA-HRP labeling (bottom) is found mainly in area 17 . Note the densest geniculocortical projection in the monocular region. Very pale transneuronal labeling is also present laterally to area 17 (up to the white arrow, area 18a).
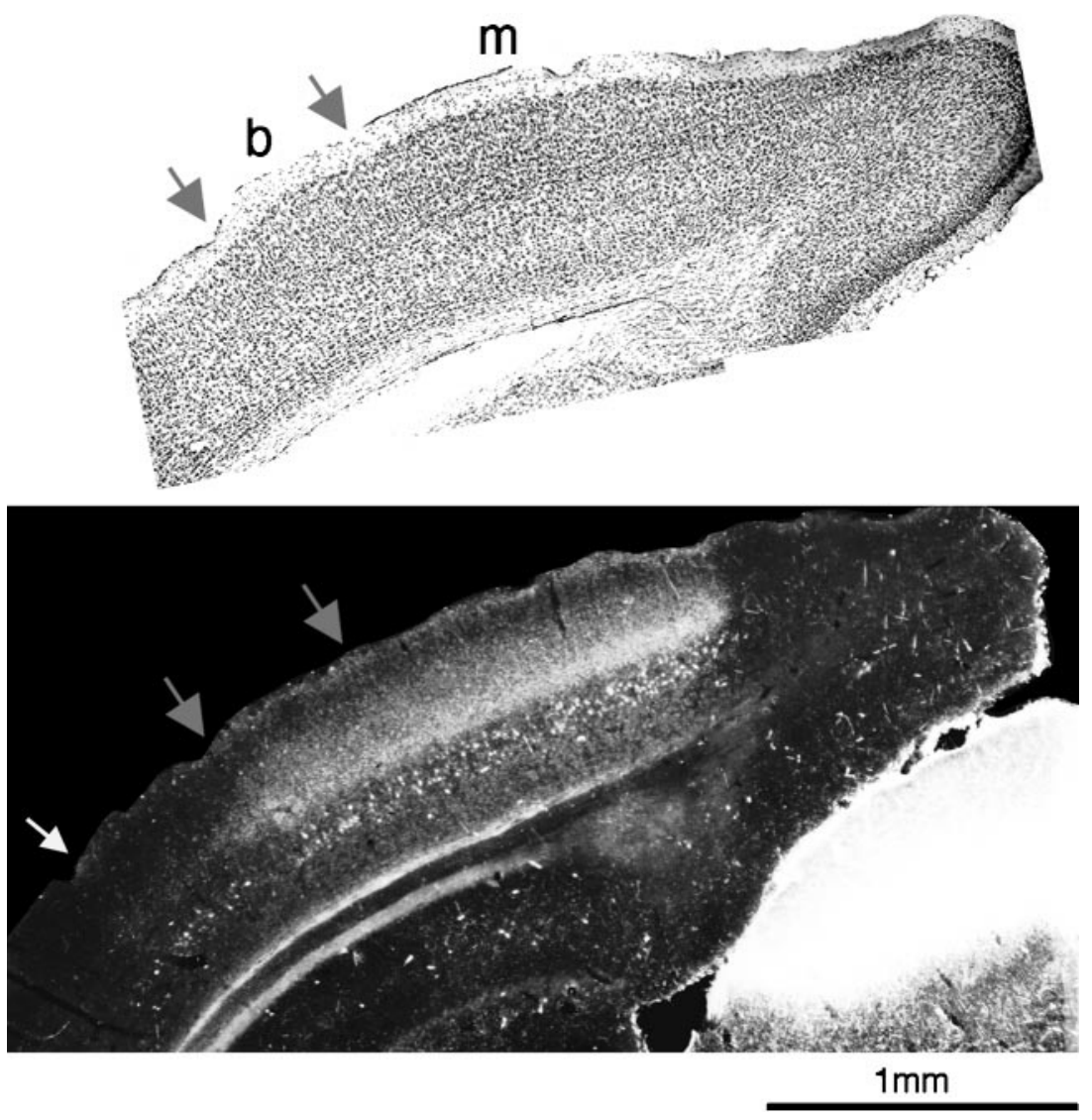

\section{cortex ipsilateral to the injected eye cortex contralateral to the injected eye}
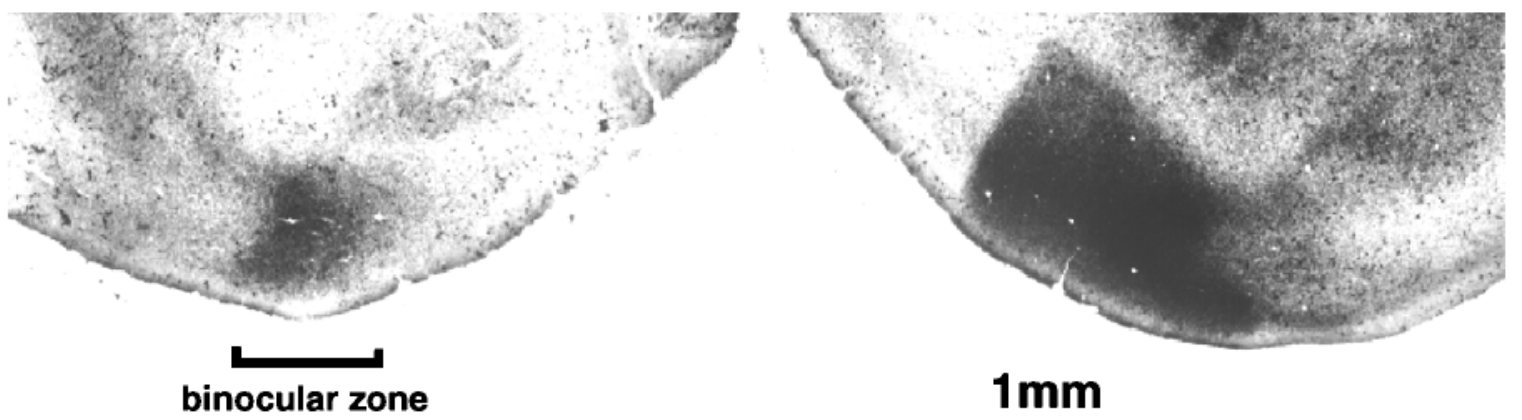

$1 \mathrm{~mm}$

Figure 4. Flattened surfaces of the posterior portion of the two hemispheres demonstrating the extent of the primary visual area as shown by the transneuronal labeling of geniculocortical terminals (dark areas) after an intraocular injection of WGA-HRP. On the side contralateral to the injected eye, the labeling is very intense, covering both the monocular and binocular regions of the visual cortex. On the side ipsilateral to the injected eye, the labeling is more restricted; its intensity and boundaries varied from animal to animal. This area of labeling defines the binocular zone. The figures are negatives of dark-field photomicrographs of a single section each.

The transneuronal labeling technique is useful for studying the anatomical characteristics and the area covered by the projection of the entire population of geniculocortical afferents. However, finer features of the projection, including changes in the size of afferent arbors, cannot be analyzed with this method. Serial reconstruction of individual afferents allows detailed analysis of this projection and the assessment of changes in arbors during development and after deprivation.

\section{Labeling and reconstruction of geniculocortical afferent arbors}

The organization of the geniculocortical pathway and its plasticity after deprivation have been studied in the visual cortex of the cat by analyzing the morphology of single geniculocortical arbors (Humphrey et al., 1985; Antonini and Stryker, 1996). We have adopted the same approach in normal and deprived mice to address three issues: (1) Do the geniculocortical inputs serving the two eyes exhibit an eye-specific pattern in the distribution of their terminal arbors? (2) Does the period of growth of geniculocortical arbors end at the end of the critical period, as delineated by the effects of brief monocular deprivation (Gordon and Stryker, 1996)? (3) Are the terminal arbors of individual afferents from the LGN to the visual cortex affected by monocular deprivation, as they are in other species, and if so, what is the nature of this effect? We focused our efforts on afferents innervating the 


\section{non-deprived projections} ipsilateral cortex
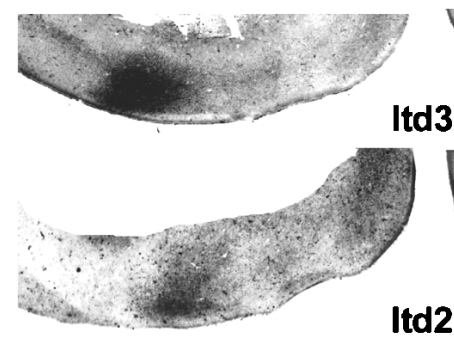

Itd2

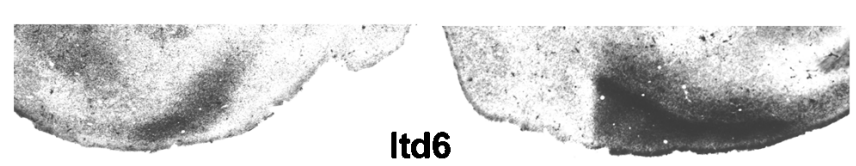

Itd6

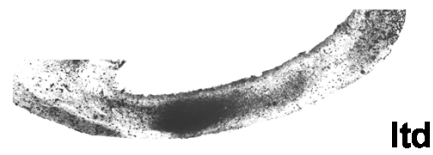

contralateral cortex
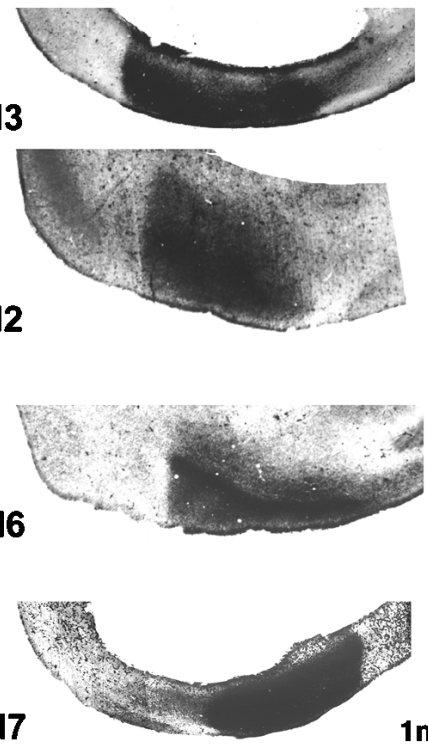

$1 \mathrm{~mm}$

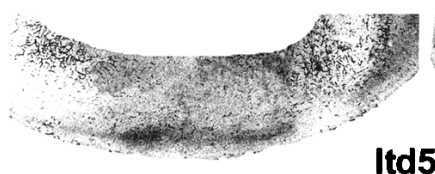

Itd8

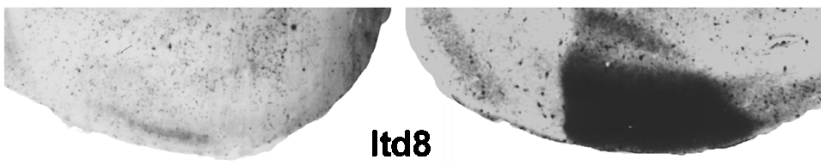

Itd5
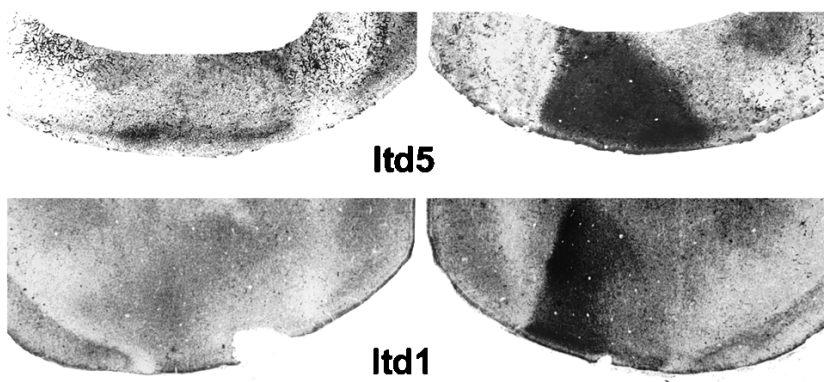

Itd1

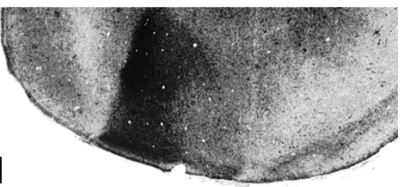

Figure 5. Tangential sections through the flattened surface of the hemispheres demonstrating transneuronal labeling of geniculocortical terminals after an injection of WGA-HRP into nondeprived eye (ltd3, ltd2, ltd6, ltd7) or deprived eye (ltd4, ltd8, ltd5, ltd1). All animals were deprived for $40 \mathrm{~d}$, ending at P60. Note in the hemisphere ipsilateral to the injected eye the strong transneuronal labeling when the nondeprived pathway was labeled and the reduced labeling when the deprived eye was injected. In contrast, a clear effect of MD on the projections serving the contralateral eye was observed only in ltd1, in which the deprived eye was injected with WGA-HRP. In this case, the lateral third of the visual cortex, presumably corresponding to the binocular zone, was less strongly labeled.

binocular portion of the visual cortex because of the physiological changes after deprivation that take place in this region.

Geniculate afferent arbors to the visual cortex were labeled anterogradely by tracer injections into the LGN and were serially reconstructed in three dimensions. Our aim was to reconstruct a homogeneous population of axon arbors. It was therefore important to consistently label axons serving one particular eye, and the identification of the eye of origin of the labeled arbors was further essential in assessing the effects of monocular deprivation. Because the portion of the LGN receiving projections from the ipsilateral eye is quite small and often contains fibers of passage from the contralateral retina, a tracer injection aimed at neurons receiving input exclusively from the ipsilateral eye is technically difficult. Therefore, we chose to make injections of the tracer into the dorsal portion of the LGN, which contains neurons responding exclusively to the contralateral eye.

Biocytin or Pha-L tracer injections for labeling geniculocortical afferents were made at the dorsal border of the anterior half of the LGN, which contains the representation of the binocular portion of the upper visual field. In all cases, the site of injection had been physiologically characterized by single- or multi-unit extracellular recordings through the pipette containing the tracer. The published data on the topographic organization of the rat LGN (Reese and Cowey, 1983; Reese and Jeffrey, 1983; Reese, 1988) were useful in guiding the electrode penetrations in this region. The lamination of the LGN was revealed by injection of fluorescent dextran into one eye, allowing verification in all deprived animals that the tracer injection was completely confined to the contralateral eye's representation. Figure $6 A$ shows a series of coronal sections through the anteroposterior extent of the LGN revealing the portions of the nucleus that receive axons from the contralateral and ipsilateral eyes. Figure $6 B$ shows an example of a biocytin injection site and its relation to the retinal terminations, shown by montage of the fluorescence label from the same section. In all cases the dorsolateral margins of the injection site reached the edge of the nucleus, and there was no overlap with the ipsilateral eye's representation as revealed by the fluorescent label.

Geniculocortical afferents were well labeled by both tracers, as shown for biocytin in Figure 7. The densest labeling was found in layer IV. However, the superficial layers also received a strong LGN projection, and often, axonal branches ran 100-200 $\mu \mathrm{m}$ tangentially below the pial surface (Fig. $7 B$ ). Ramifications in layers V and VI were usually sparse. Afferents were distributed homogeneously in the area of labeling, and there was no sign of subdivision into eye-specific domains such as the ocular dominance patches in higher mammals. Arbors were chosen for reconstruction in zones of relatively sparse labeling, as shown in Figure 7, $B$ and $C$.

A few labeled geniculocortical afferents were consistently found to arise from the white matter and arborize 300-700 $\mu \mathrm{m}$ lateral to the lateral edge of the dense area of labeling. This region is most probably area $18 \mathrm{a}$, although we could not verify it on the basis of cytoarchitectonic features, because the reaction products of the biocytin prevented a good Nissl counterstaining. The presence of a few geniculocortical afferents in area 18a is in agreement with the light labeling observed in the transneuronal experiments (Fig. 3). Branches from axons arborizing within area 17 were not seen to extend into this lateral region, except in a single case (Fig. 8, mo $2 \mathrm{~h}$ ) in which a separate trunk arose from the white matter lateral to area 17. 


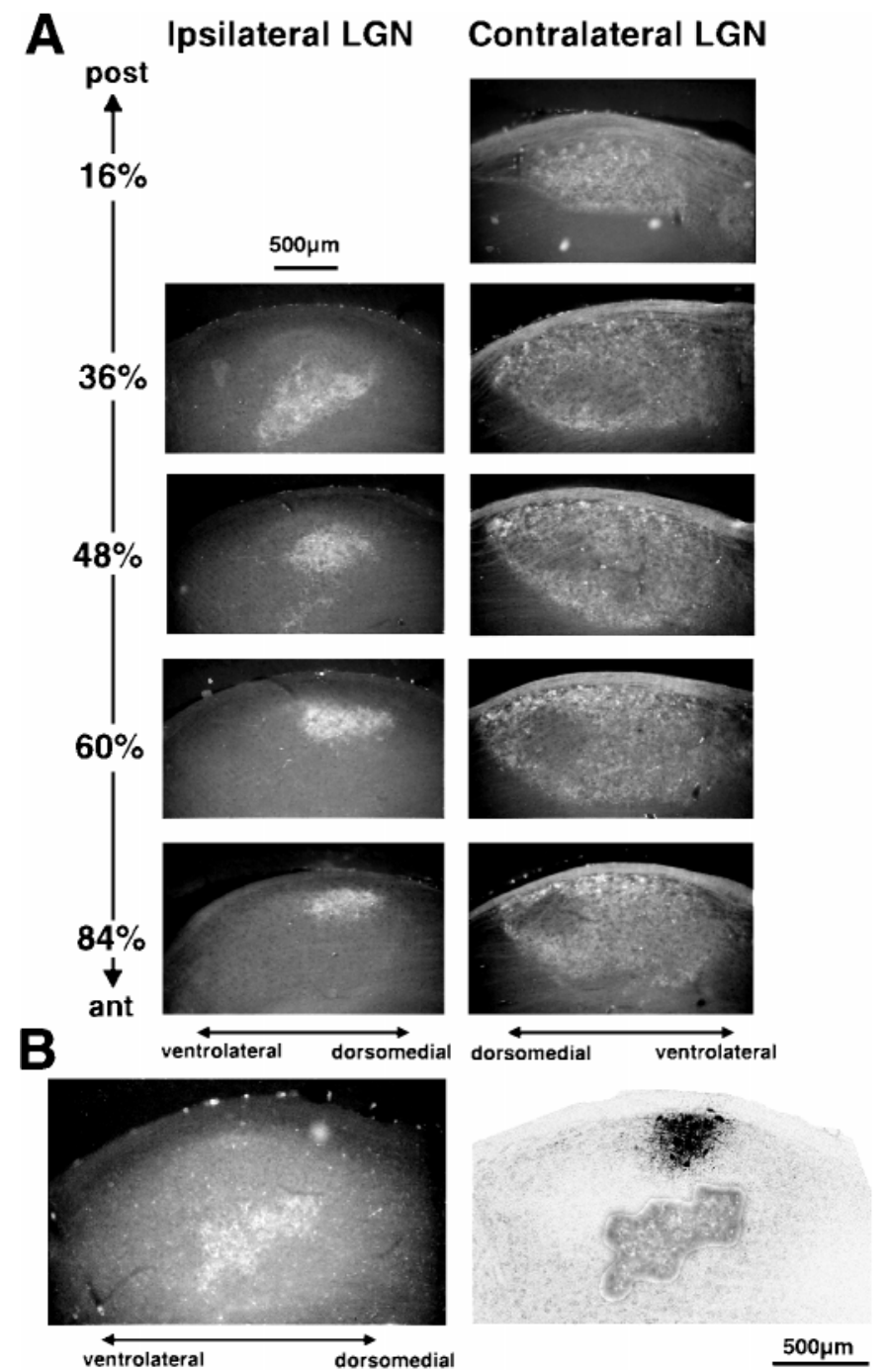

Figure 6. A, Series of anteroposterior coronal sections through the LGN contralateral and ipsilateral to a monocular injection of fluorescent dextran. The portion of the LGN receiving ipsilateral retinal fibers is confined to a small patch in the rostral half of the LGN. Dorsal is up and to the right in ipsilateral sections; $u p$ and to the left in contralateral sections. $B$, Example of a biocytin injection and its relation to the ipsilateral retinal projections. Left panel, Scan of fluorescent photomicrograph of an LGN coronal section showing the rhodamine dextran-labeled terminals arising from the ipsilateral eye. Right panel, Scan of a confocal image of the same section after biocytin histochemistry showing the biocytin injection site. The rhodamine-labeled area from the fluorescent photomicrograph has been superimposed to show that the injection site, located next to the pia in the dorsolateral portion of the LGN, did not overlap with the "ipsilateral patch."

\section{Geniculocortical afferent arbors in normal animals}

Single geniculocortical afferents were serially reconstructed in mice at two different ages, at P40 (which is at the end of the critical period of susceptibility to the effects of a brief period of monocular deprivation; Gordon and Stryker, 1996) and at P60, to determine whether the axons continued to grow and elaborate after P40 (Table 1).

Fourteen geniculocortical arbors were reconstructed in P40 animals. These arbors showed great variability in size and shape, as illustrated in Figure $8 A$. Some afferents arborized extensively and formed a dense patch of terminal branches ( $m o 2 g$ ), whereas other afferents gave rise to only a few branches (molb). Arbors

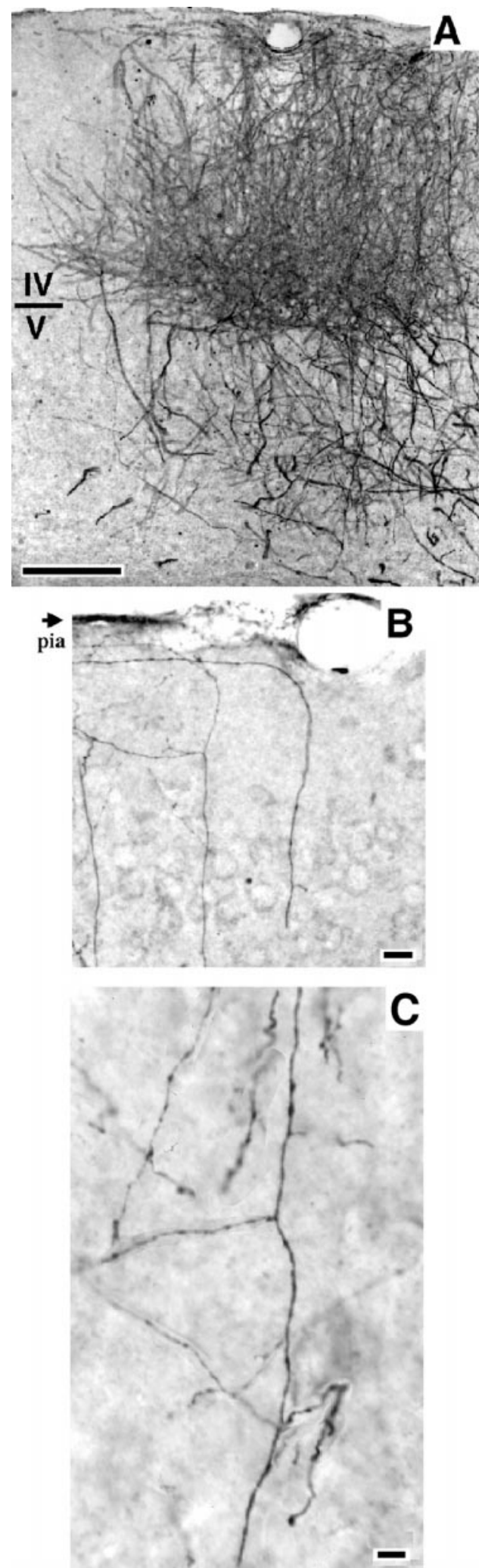

Figure 7. A, Scan of a photomicrograph of a coronal section through the visual cortex showing dense cortical labeling of biocytin-filled geniculocortical afferents. Note the abundant innervation not only of layer IV but also of the supragranular layers. $B, C$, Branches of biocytin-labeled geniculocortical arbors presented as a collage of photomicrographs combining serial focal planes. Note in $B$ the ramifications running beneath the pial surface. Note in $C$ branches running in layer IV. Scale bars: $A, 200$ $\mu \mathrm{m} ; B, 50 \mu \mathrm{m} ; C, 20 \mu \mathrm{m}$. 


\section{A P40 Normal Geniculocortical Arbors}
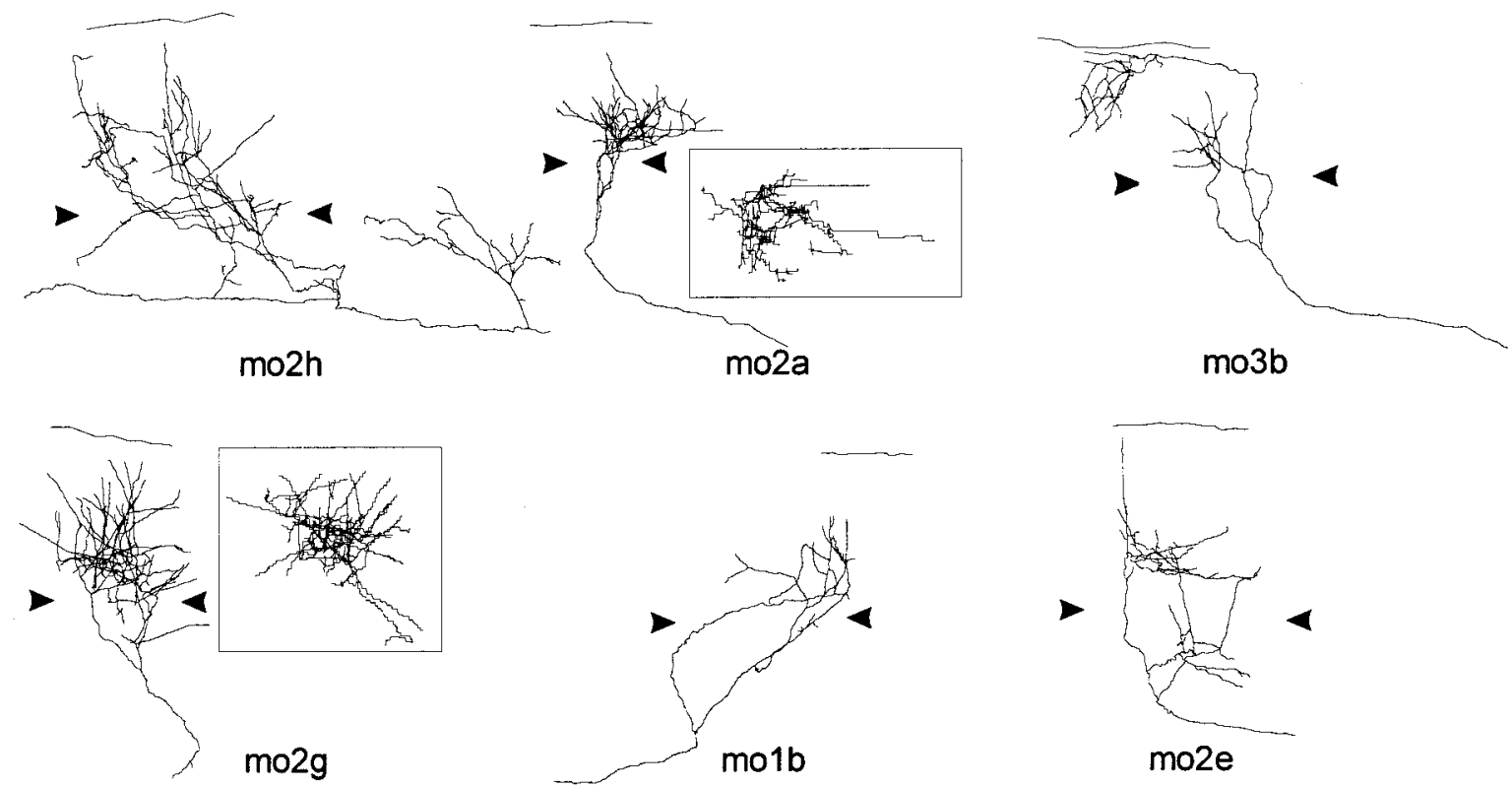

\section{B P60 Normal Geniculocortical Arbors}

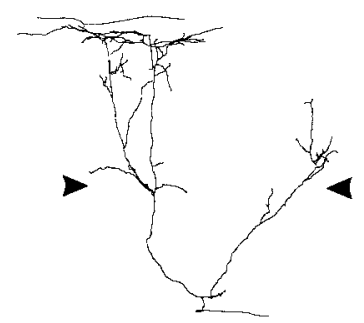

mo4a

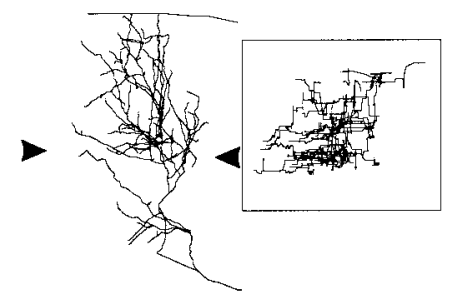

mo4b

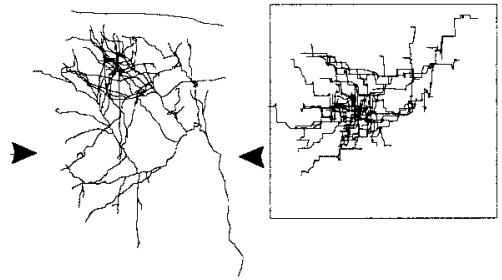

mo4c

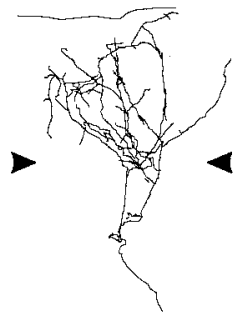

$\operatorname{mo4d}$

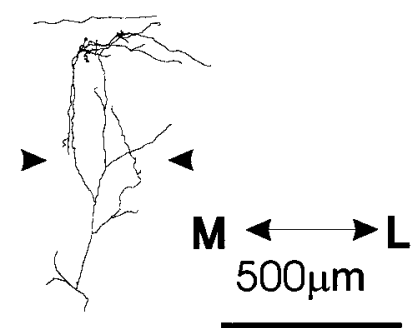

mo4e

Figure 8. Computer reconstructions of geniculocortical arbors in area 17 comparing normal $\mathrm{P} 40(A)$ and $\mathrm{P} 60(B)$ mice. The arrowheads indicate the boundary of layer IV. All arbors are presented in coronal view, and the most complex arbors are also presented in surface view after a $90^{\circ}$ rotation along an axis passing through layer IV (mo2a, mo2g, mo4b, mo4c, insets). The line above coronal views indicates the pial surface. The scale is for all arbors.

differed also in the laminar pattern of arborization. In most arbors the majority of collaterals resided in layer IV, with a few branches extending into the superficial layers and layers V and VI (mo2a, mo2g). In two arbors, the predominant arborization was in layer I. One example, $m o 3 b$, is shown in Figure $8 A$. Extensive ramifications in the infragranular layers were also found in six arbors, examples of which are shown in Figure $8 A$ (mo2h, mo2e).
On average, the extent of layer IV covered by a single geniculocortical afferent was $493 \mu \mathrm{m}$ (range, 200-1700 $\mu \mathrm{m}$ ) in the mediolateral axis and $395 \mu \mathrm{m}$ (range, 50-640 $\mu \mathrm{m}$ ) in the anteroposterior axis.

The five geniculocortical arbors reconstructed at P60 showed the same variability that was present at P40 (Fig. $8 B$ ). Three afferents arborized densely in layers IV and III (Fig. $8 B$, mo $4 b$, 
$m o 4 c, m o 4 d$ ); the remaining two were rather sparsely ramified with most branches located in the superficial layers (Fig. 8B, mo4a, mo4e). The mean extension of the terminal arborization was $581 \mu \mathrm{m}$ along the mediolateral axis (range, 370-960 $\mu \mathrm{m}$ ) and $408 \mu \mathrm{m}$ along anteroposterior axis (range, 280-480 $\mu \mathrm{m}$ ). At both ages, the terminal fields of geniculocortical arbors were not organized in distinct patches.

Quantification of the total length and number of branch points of the portion of the afferents ramifying both in layer IV and supragranular layers indicates that arbors at P60 were larger and more complex than arbors at P40 (total length, $p<0.02$; branch points, $p<0.03$ ), suggesting continued growth between these two ages. As illustrated in Figure 11, $A$ and $B$, the total length of 8 of 14 arbors at $\mathrm{P} 40$ was $<6000 \mu \mathrm{m}$, whereas the smallest arbor at $\mathrm{P} 60$ had a total length of $6100 \mu \mathrm{m}$. Moreover, 12 of 14 arbors at P40 had $<40$ branch points, whereas only 1 of the 5 arbors reconstructed at P60 had so few. The two groups did not differ in their mediolateral or anteroposterior extents.

In summary, geniculocortical arbors in normal mice are very heterogeneous, and their growth continues after the peak of the critical period.

\section{Geniculocortical afferent arbors in monocularly deprived animals}

The aim of this series of experiments was to determine whether the physiological effects of MD (Dräger, 1978; Gordon and Stryker, 1996) can be ascribed to changes in the morphology of single geniculocortical axons. Animals were deprived from P20 (the beginning of the critical period; Gordon and Stryker, 1996) for 17-19 or 36-44 d and were perfused at P37-P39 or P56-P64. The deprivations ending around $\mathrm{P} 40$ were designed to examine anatomical plasticity around the peak of the critical period for the physiological effects of deprivation. Because we observed continued growth of the afferents in normal animals after this time, we also examined more prolonged deprivations ending at approximately P60 to determine whether anatomical plasticity induced by MD accompanied this continued growth. For technical reasons explained above, we analyzed only geniculocortical afferents serving the contralateral eye. Table 1 lists the animals used in this series of experiments, the deprivation protocol, and the arbors reconstructed in each group.

\section{P40 animals monocularly deprived for $20 d$ around the peak of the critical period}

Six arbors serving the deprived eye (Fig. 9A) and six arbors serving the nondeprived eye (Fig. 9B) were reconstructed in animals deprived for $20 \mathrm{~d}$ and perfused at P40. Both groups of arbors had patterns of cortical innervation similar to arbors reconstructed in normal age-matched controls. For example, axon collaterals were found in the superficial layers, including layer I, as well as in layer IV.

Two of the deprived arbors had a rather dense arborization in layer IV (Fig. 9A, $d 5 d a, d 8 d b$ ). One arbor (Fig. 9 $A, d 3 d b$ ) ramified in both the supragranular and infragranular layers but not in layer IV. The remaining two arbors had very narrow and poorly ramified terminal fields (Fig. $9 A, d 5 d b, d 8 d a$ ). On average, the extension of the arbors in layer IV and the supragranular layers was 491 $\mu \mathrm{m}$ (range, 200-1060 $\mu \mathrm{m}$ ) in the mediolateral axis and $547 \mu \mathrm{m}$ (range, 240-880 $\mu \mathrm{m}$ ) in the anteroposterior axis.

As a group, arbors serving the nondeprived eye (Fig. 9B) were more homogeneous than both normal and deprived arbors. With one exception (Fig. 9B, $d 7 n d c$ ), these arbors qualitatively ap- peared to be as richly ramified as the densest arbors reconstructed in normal animals. The average extension of the terminal arborization was $388 \mu \mathrm{m}$ along the mediolateral axis (range, 300-520 $\mu \mathrm{m}$ ) and $400 \mu \mathrm{m}$ along anteroposterior axis (range, 240-600 $\mu \mathrm{m}$ ).

The scattergrams in Figure 11 provide quantification of the total length (see Fig. 11 $A$ ) and number of branch points (see Fig. $11 B$ ) for arbors reconstructed in normal animals and for both deprived and nondeprived arbors reconstructed in MD animals. The mean value of each group is also plotted in the same graph. In P40 animals, no statistical differences were found in either total length or number of branch points between deprived arbors and arbors reconstructed in normal, age-matched controls. Moreover, although on average the nondeprived arbors were longer (1.8 and 1.4 times, respectively) and more ramified (1.8 and 1.7 times, respectively) than both deprived and normal arbors, because of arbor variability only the differences between nondeprived and normal arbors was significant ( $p<0.05$ for both parameters). Deprived and nondeprived arbors did not differ in either mediolateral or anteroposterior extensions, and both groups were not different from normal arbors.

\section{P60 animals monocularly deprived for $40 \mathrm{~d}$}

Seven geniculocortical arbors serving the deprived eye (Fig. 10A) and six arbors serving the nondeprived eye (Fig. 10B) were reconstructed in P60 animals monocularly deprived for $40 \mathrm{~d}$. Both groups of arbors exhibited similar variability in size and complexity as normal arbors reconstructed at P60. All arbors, deprived and nondeprived, had their main arborization in layer IV. Three deprived arbors (Fig. 10 $A, d 4 d a, d 4 d b, d 4 d d$ ) extended collateral branches toward layer I, as did, with one exception (Fig. 10B, $d(n d b)$, all nondeprived arbors.

For the deprived arbors, the mean extension of the terminal arborization was $401 \mu \mathrm{m}$ along the mediolateral axis (range, $350-655 \mu \mathrm{m}$ ) and $405 \mu \mathrm{m}$ along anteroposterior axis (range, $200-640 \mu \mathrm{m})$. For the nondeprived arbors, the mean extension of the terminal arborization was $588 \mu \mathrm{m}$ along the mediolateral axis (range, 260-800 $\mu \mathrm{m}$ ) and $693 \mu \mathrm{m}$ along anteroposterior axis (range, 520-1000 $\mu \mathrm{m}$ ). The nondeprived arbors differed significantly from the deprived arbors only in the anteroposterior extension $(p<0.02)$.

The quantification of the total length and number of branch points for deprived and nondeprived arbors at P60, along with results from $\mathrm{P} 60$ normal arbors, is shown in the scattergram of Figure 11, $A$ and $B$. The mean total length of nondeprived and normal arbors was 1.6 times larger than that of deprived arbors. Deprived arbors were statistically different from nondeprived arbors in total length $(p<0.03)$, and the difference from normal arbors was close to significant $(p=0.06)$. Moreover, deprived arbors at P60 were similar in length and complexity to deprived arbors at P40 $(p>0.7)$, indicating a lack of growth after P40. Nondeprived arbors did not differ statistically from normal arbors at P60.

Even in normal mice, single geniculocortical arbors serving the contralateral eye are heterogeneous in many aspects, including length, complexity, and pattern of projection. This heterogeneity masks to some extent the effects of deprivation and prevents some of the differences between deprived and nondeprived arbors from reaching statistical significance.

An overall picture of the significant changes during development and plasticity of the geniculocortical arbors is illustrated in Figure 11C. For each group of geniculocortical arbors (normal, deprived, and nondeprived at P40 and P60), the mean values for 


\section{A P40 Deprived Geniculocortical Arbors}

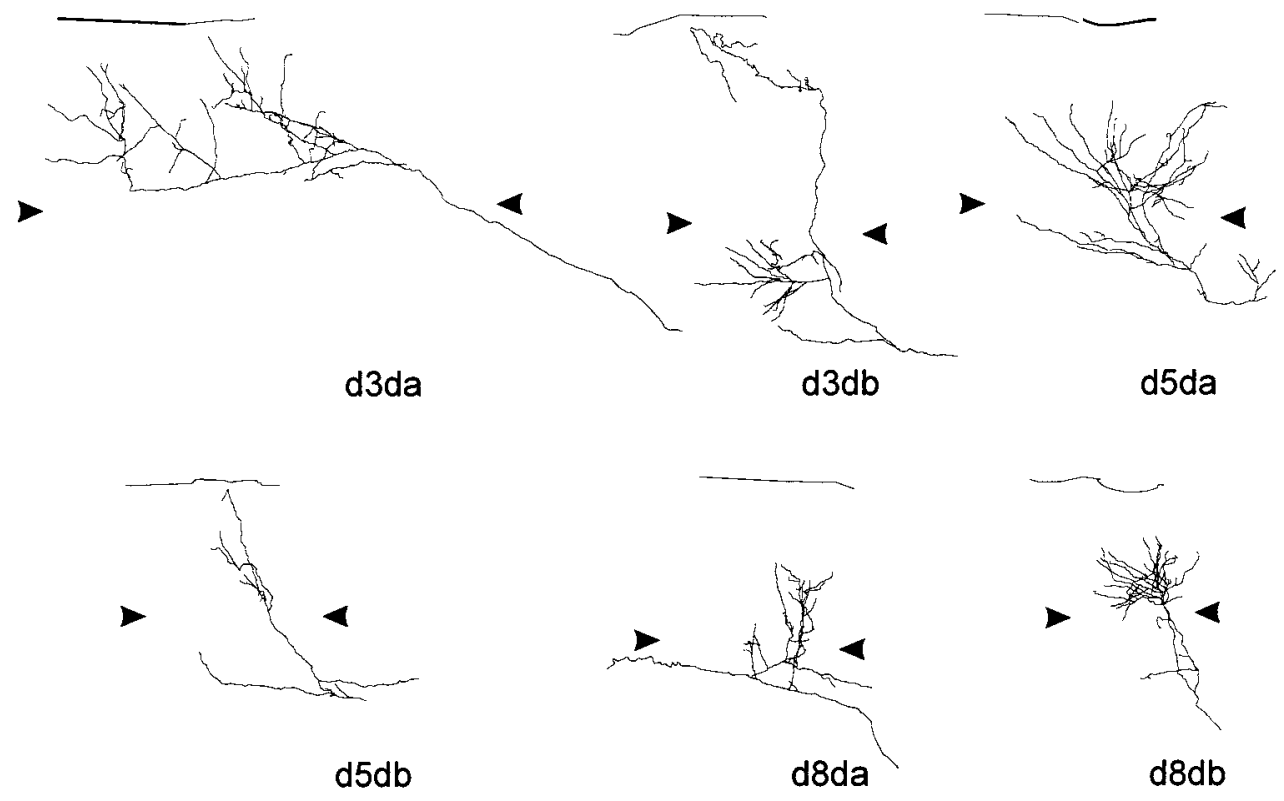

\section{B P40 Non-Deprived Geniculocortical Arbors}

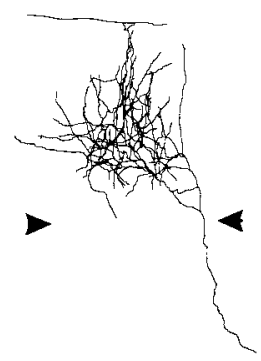

d2nda

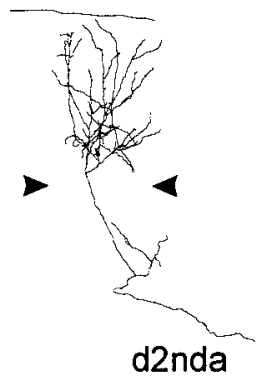

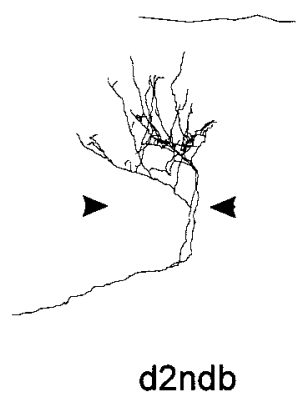

$\mathrm{d} 2 \mathrm{ndb}$

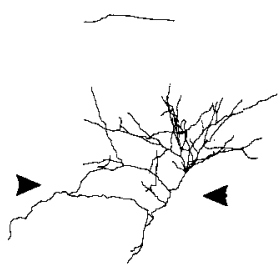

d7ndb

\section{$M$} $500 \mu \mathrm{m}$

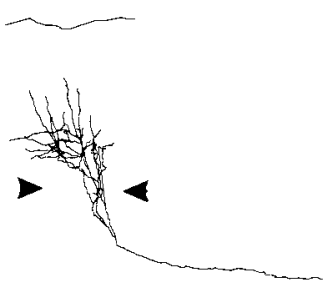

d2ndc total length and number of branch points have been normalized relative to the mean values of normal arbors at $\mathrm{P} 40$. These parameters changed in parallel. The graph shows the significant growth of normal arbors between the age of P40 and P60. It shows also that $20 \mathrm{~d}$ of MD tends to allow the nondeprived arbors to become significantly larger and more complex than normal, whereas the deprived arbors are not modified. When the deprivation is maintained for $40 \mathrm{~d}$, these deprived arbors exhibit only slight and insignificant further growth.

\section{Physiological analysis of the visual cortex in normal} and monocularly deprived mice

Intrinsic signal optical imaging

Intrinsic signal optical imaging has been used in many species to reveal both the spatial distribution and magnitude of responses to different stimuli in the visual cortex, and it has been used to map orientation columns, ocular dominance columns, and other features of normal cortical organization (Bonhoeffer and Grinvald, 


\section{A P60 Deprived Geniculocortical Arbors}
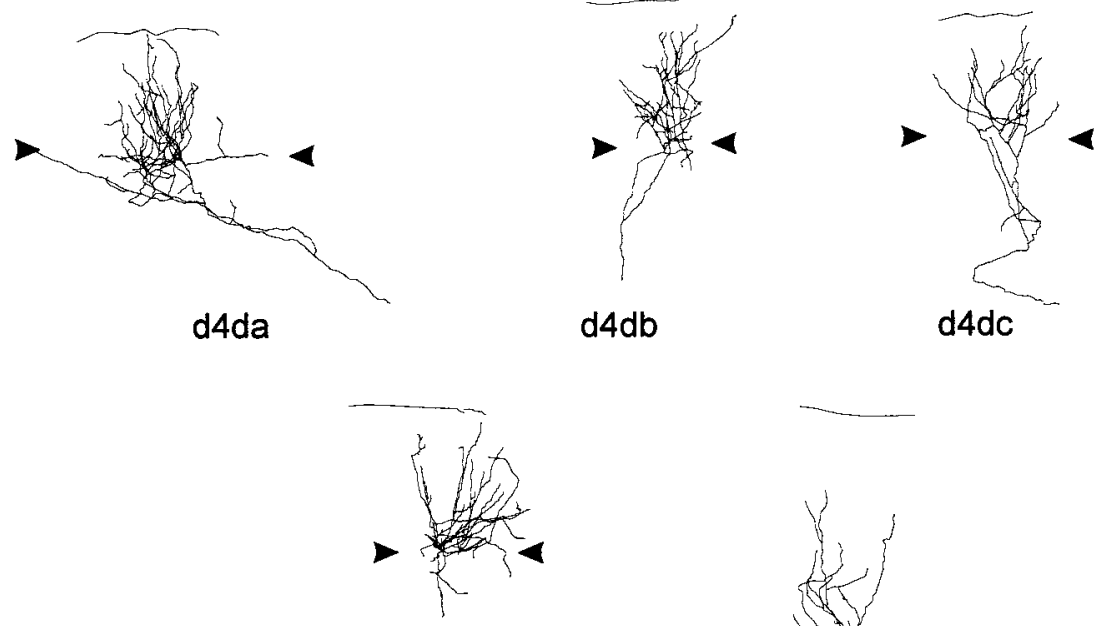

d4dd

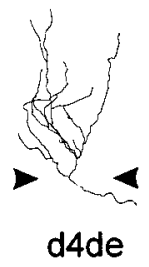

B P60 Non-Deprived Geniculocortical Arbors

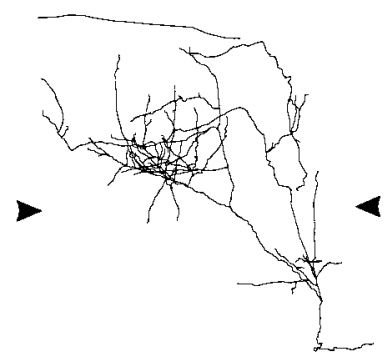

d1nda

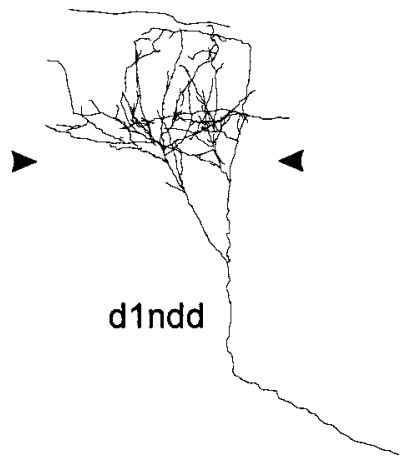

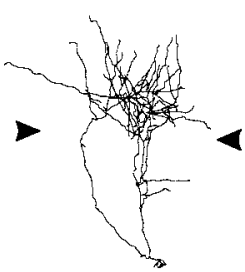

$d 1 n d b$

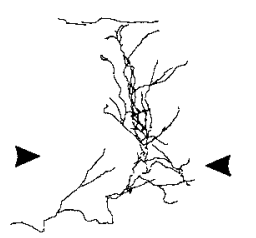

d6nda

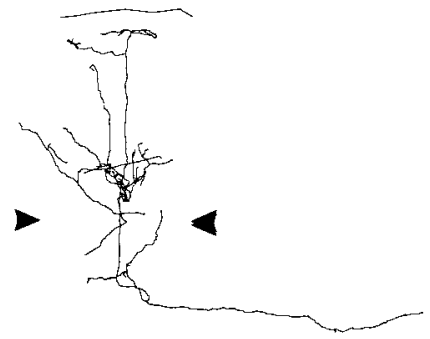

d1ndc

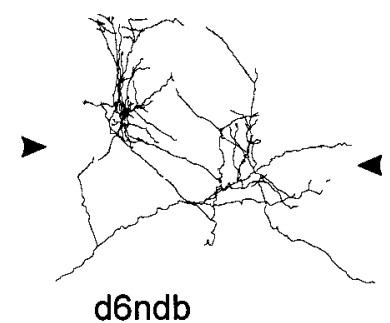

$500 \mu \mathrm{m}$

Figure 10. Single, serially reconstructed geniculocortical arbors in area 17 in P60 animals monocularly deprived for 40 d starting at P20. A, Arbors serving the deprived eye. $B$, Arbors serving the nondeprived eye. The arrowheads indicate the boundary of layer IV. The scale is for both groups of arbors.

1993). We sought to determine whether this physiological approach would be useful in the study of the visual cortex of the mouse. Using techniques that routinely reveal visual cortical organization in the cat in our laboratory (Crair et al., 1997), we stimulated the two eyes of the mouse separately with drifting low spatial frequency gratings of different orientations (see Materials and Methods for details). Figure 12, $A$ and $B$, shows that strong optical signals were produced in the visual cortex of a normal P35 mouse in response to stimulation of the two eyes. Similar results were obtained in three additional normal mice, and responses to the different stimulus orientations in all animals were similar to one another and failed to reveal the presence of orientation 


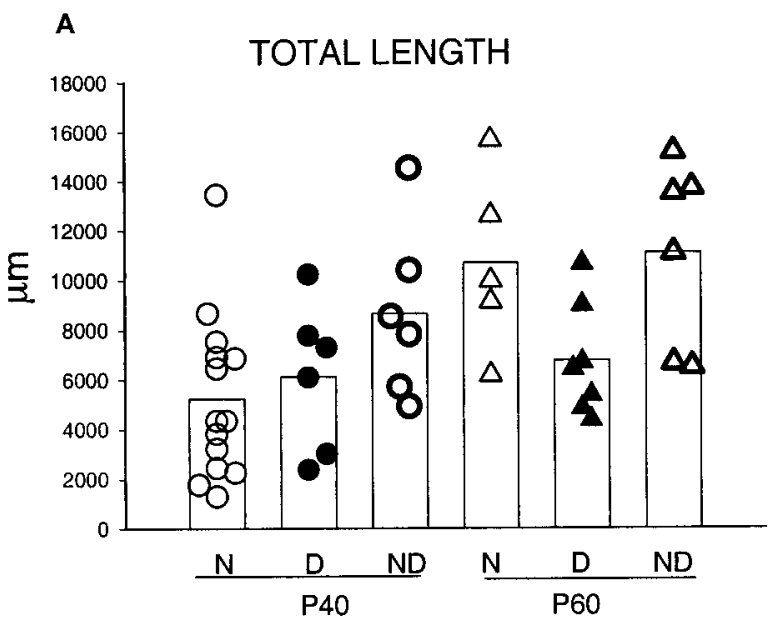

B
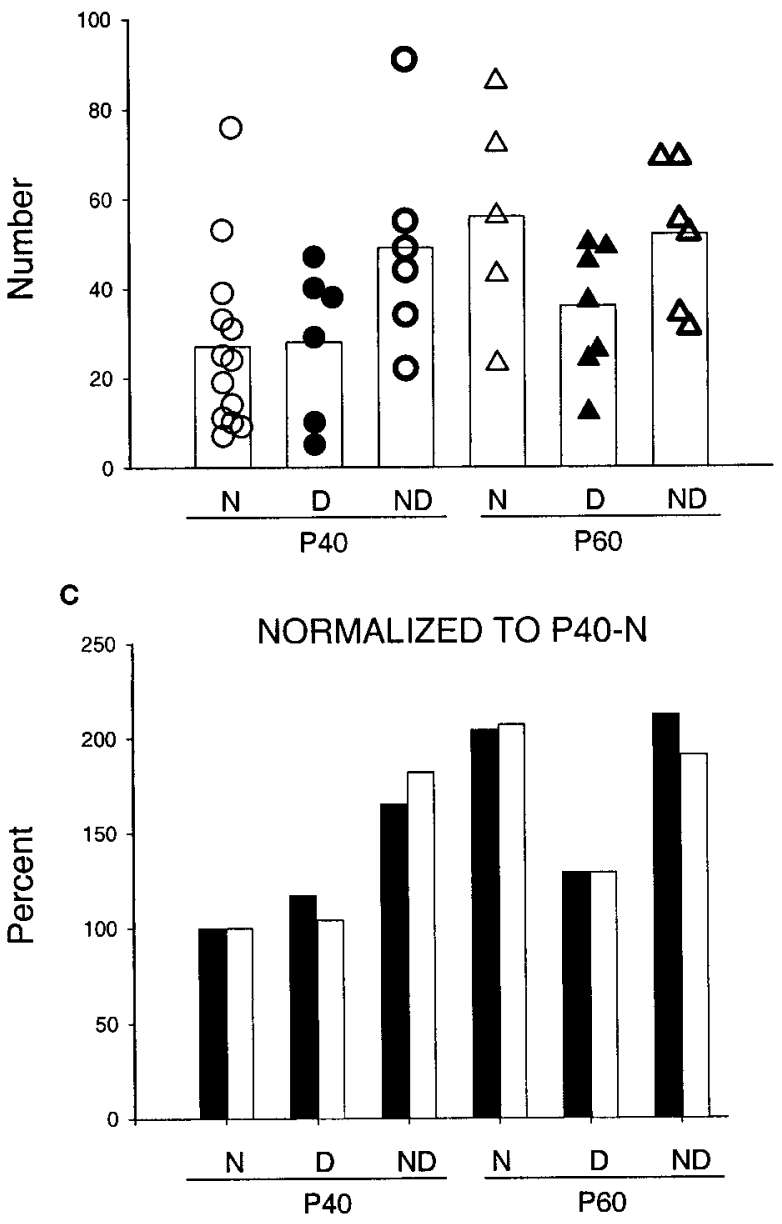

Figure 11. Scattergrams of the total length $(A)$ and number of branch points $(B)$ for arbors reconstructed in normal animals $(N)$ and for both deprived $(D)$ and nondeprived $(N D)$ arbors reconstructed in MD animals. The age at perfusion is also indicated (P40 and P60). The white bar represents the mean value in each group. $C$, Values of total length ( filled bars) and number of branch points (open bars) of arbors in the six experimental groups normalized relative to the mean values of arbors in normal arbors at P40. columns (data not shown). Comparing optical responses to the two eyes in the two hemispheres clearly revealed the monocular and binocular zones within the primary visual cortex. Figure $12 B$ shows that stimulation of the ipsilateral eye produced a rostrocaudal band of activation (dark) that occupies the lateral portion of the region activated by the contralateral eye (Fig. 12A). The image of the ratio between activation by the two eyes (Fig. 12C) reveals the monocular zone as a dark rostrocaudally elongated area and, just medial to it, the binocular zone in which activation by the contralateral eye is only slightly stronger than by the ipsilateral eye. Single-unit recordings at the positions indicated by the stars in Figure $12 A-D$ confirmed the conclusion that the band of ipsilateral activation represents the binocular segment of primary visual cortex and that the area medial to this was activated exclusively by the contralateral eye. No finer subdivision of responses to the two eyes within the binocular zone was clear, consistent with the failure to see ocular dominance patches in the anatomical experiments described above.

Because optical imaging in normal mice clearly revealed the overall cortical responses to each eye, we studied mice that had been monocularly deprived to determine whether the physiological effects of monocular deprivation would be evident. Figure 12, $E, F, I$, and $J$, shows optical responses to stimulation of the two eyes in both hemispheres of a P40 mouse that had been monocularly deprived for $20 \mathrm{~d}$. In the hemisphere ipsilateral to the deprived eye, responses to the deprived (ipsilateral) eye are nearly absent (Fig. 12F), whereas the nondeprived (contralateral) eye activates the cortex strongly (Fig. 12E). The ocular dominance ratio map shows a dark oval region of contralateral dominance encompassing both monocular and binocular zones of area 17 (Fig. 12G). In the hemisphere contralateral to the deprived eye, responses to the nondeprived (ipsilateral) eye are strong (Fig. $12 J)$, much stronger than in normal animals, whereas responses to the deprived (contralateral) eye are weaker than normal, particularly in the binocular zone (Fig. 12I). The ocular dominance ratio map (Fig. $12 K$ ) reveals most prominently a nearly white area, indicating dominance by the nondeprived ipsilateral eye over the binocular zone, and shows a darker region, indicating activation by the deprived eye, only in the monocular segment. Similar results were obtained in three other animals (data not shown). In summary, optical imaging revealed consistent changes in the response of the ipsilateral eye's pathways after monocular deprivation and in the relative responses of the two eyes within the binocular zone. Effects of deprivation on the contralateral eye's responses in the monocular zone were seen in some cases, particularly around its edges, but were inconsistent. Intrinsic signal optical imaging provides a reliable method for investigating responses in the visual cortex of the mouse.

\section{Single-unit recordings}

The critical period of susceptibility to the effects of monocular deprivation in the mouse has been defined using brief periods of eyelid suture. The critical period defined with $4 \mathrm{~d}$ eye closure peaks at approximately P26 and declines sharply thereafter, with little or no effect of such brief deprivation evident after P36 (Gordon and Stryker, 1996). Our anatomical experiments showed that geniculocortical afferent arbors continue to grow from P40 to P60, well beyond the critical period as defined above. In addition, the effects of MD on single afferent arbors and on the whole geniculocortical projection labeled transneuronally become more pronounced when monocular deprivation is prolonged up to P60. Therefore, we sought to analyze whether the physiological effects 

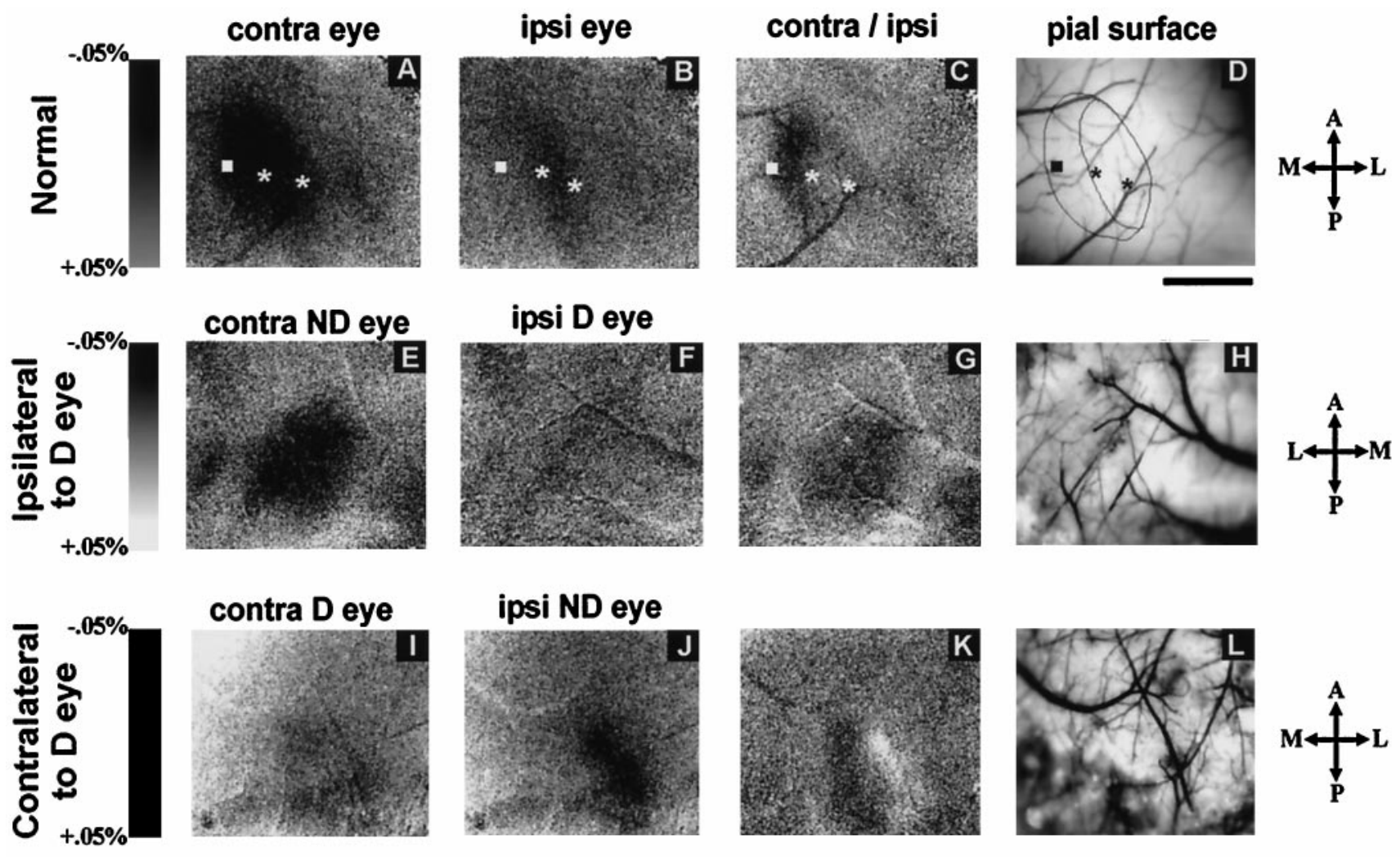

Figure 12. Intrinsic signal optical responses in mouse visual cortex. $A-D$, Normal mouse. $E-H$, Hemisphere ipsilateral to deprived eye in MD mouse. $I-L$, Hemisphere contralateral to deprived eye in MD mouse. Darkness indicates response to visual stimulation as percent change in reflectance as on the scale to the left of each row. $A, E, I$, Responses elicited from eye contralateral (contra) to the hemisphere imaged. $B, F, J$, Responses elicited from eye ipsilateral (ipsi) to the hemisphere imaged on scales identical to $A, E$, and $I$. $C, G, K$, Ratio between activation by contralateral and ipsilateral eyes, with darkness indicating greater response to contralateral and lightness indicating greater response to ipsilateral eye. $D, H, L$, Images of blood vessels on the cortical surface aligned with optical maps in the same row. Monocular and binocular zones of cortex are outlined in a normal case $(D)$, along with electrode penetration sites. Receptive fields of neurons recorded at the leftmost site were in the monocular segment of the visual field; receptive fields at two sites to the right were located successively more central and in the binocular visual field. Note the virtual disappearance of the deprived eye responses in the ipsilateral hemisphere $(F)$ and the increase in the response to the nondeprived eye in its ipsilateral hemisphere $(J)$. Note also the reduction in the deprived eye's response in its contralateral hemisphere $(I)$. Length scale, $1 \mathrm{~mm}$ for all images. Rostral is up and caudal is down in all figures. Medial is left in $A-D$ and $I-M$, whereas medial is right in $E-H$. Lateral is right in $A-D$ and $I-M$, whereas lateral is left in $E-H$.

of prolonged deprivation might also reveal continued plasticity beyond the end of the critical period as defined above. The overall ocular dominance of the binocular segment of the visual cortex was measured from the responses of 20-31 single units recorded in three or four vertical penetrations spaced $200 \mu \mathrm{m}$ apart from each other in the superficial layers and was summarized by both the seven-classes ocular dominance distribution (Hubel and Wiesel, 1962) and the CBI defined previously (and see Materials and Methods). Figure 13, $A$ and $B$, shows that the overall dominance of the contralateral eye was confirmed in five normal animals between P45 and P60, with a mean CBI of 0.75 (range, 0.73-0.78). After MD, we made recordings in the hemisphere contralateral to the deprived eye. In three animals deprived from P20 to P40, the effects of $20 \mathrm{~d}$ of contralateral eye deprivation were similar to those previously reported for $4 \mathrm{~d} \mathrm{MD}$, a mean CBI of 0.45 , indicating a substantial shift of response in favor of the open, ipsilateral eye (Fig. 13A,C). When the deprivation was further prolonged, extending from P20 to P60, its effect was somewhat greater: a mean CBI of 0.39 , consistent with a residual plasticity after P40 (Fig. 13A,D). We therefore deprived another group of four mice for $20 \mathrm{~d}$ beginning at $\mathrm{P} 40$ and found a mild but consistent effect of deprivation, yielding a mean CBI of 0.61 , significantly ( $p<0.03$, unpaired $t$ test) below that of normal animals of similar age (Fig. 13A,E). These physiological results suggest that a small but significant plasticity persists beyond $\mathrm{P} 40$ though the period up to P60 during which geniculocortical afferents continue to grow.

Both physiological approaches, optical imaging and single units, reveal strong plasticity during the period before P40.

\section{DISCUSSION}

The experiments described in this paper were directed toward understanding the anatomical changes underlying functional plasticity in the developing visual cortex of the mouse. We begin by describing the location of binocular and monocular segments of the visual cortex in normal histological preparations of the mouse. We then examine geniculocortical input labeled transneuronally and at the level of individual afferent arbors in normal and monocularly deprived animals. Finally, we compare the anatomical plasticity to two different physiological measures of visual responses: intrinsic signal optical imaging and extracellular microelectrode recording.

In normal animals, transneuronal label after an eye injection clearly delineated the monocular and binocular zones of area 17, with no spread across a sharp border shared with area $18 \mathrm{~b}$ on the medial side but a slight invasion of area 18a laterally. Optical 
A
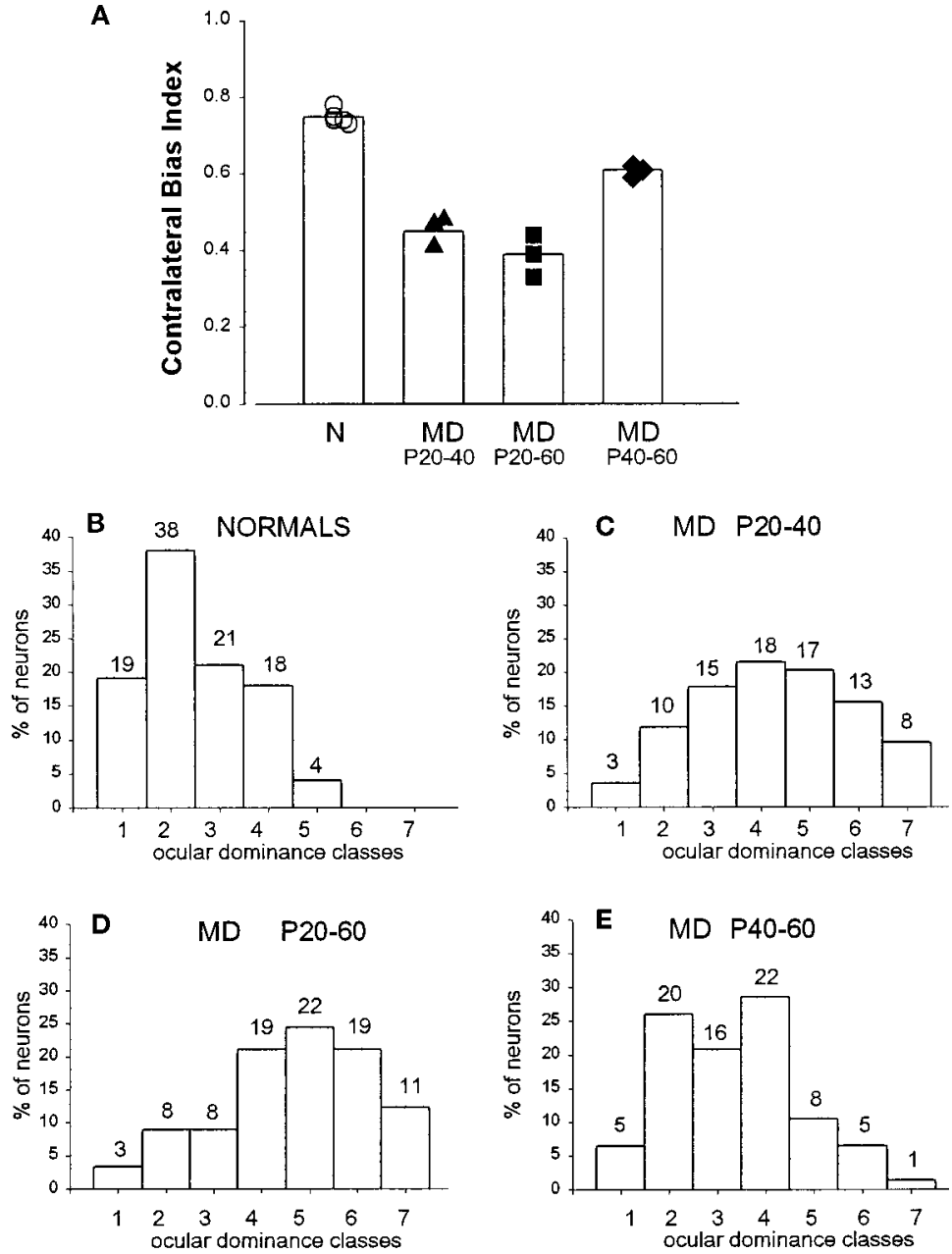

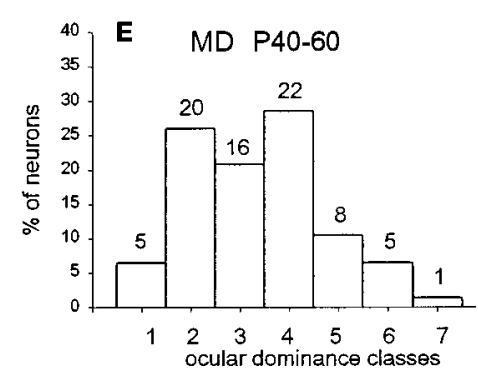

Figure 13. CBI (mean and individual values) and ocular dominance in normal and monocularly deprived animals after different deprivation protocols. $A$, Single neuron responses in normal mice are dominated by the contralateral eye (mean $\mathrm{CBI}=0.75)$. After monocular deprivation, CBIs in the visual cortex ipsilateral to the open eye decrease to 0.45 and 0.39 after 20 and $40 \mathrm{~d}$ of MD, respectively, indicating dominance of the ipsilateral eye. Late MD, from P40 to 60, is still able to affect the eye dominance of visual cortical neurons. $B-E$, Percent of cells assigned to each of the seven ocular dominance classes (Hubel and Wiesel, 1962) in normal animals and in animals monocularly deprived from P20 to P40, from P20 to P60, and from P40 to P60, respectively. The number on top of each ocular dominance class indicates the actual number of cells. imaging also showed monocular and binocular zones but revealed no finer organization of ocular dominance or orientation selectivity. Single geniculocortical afferents serving the contralateral eye and innervating the binocular segment of area 17 showed great heterogeneity in length, complexity, and pattern of cortical projections. Many individual arbors were large enough nearly to cover the mediolateral extent of the binocular portion of the visual cortex, and they showed no clustering consistent with the presence of ocular dominance patches. Growth and elaboration of terminal arbors continue from P40 to P60, significantly beyond the peak of the critical period (at approximately P26; Gordon and Stryker, 1996).

Monocular deprivation ending at P40 appeared to promote the growth of the open eye's contralateral projection without causing the closed eye's contralateral input to shrink. Continued deprivation to P60 prevented the growth of the closed eye's contralateral inputs. A continuation of functional plasticity between P40 and P60 was found in microelectrode studies, and transneuronal labeling showed that the ipsilateral eye's projection was severely affected by prolonged deprivation to P60. Functional imaging showed profound effects of deprivation, particularly in the ipsilateral pathway, even at P40.

\section{The primary visual cortex and its geniculate input in the normal animal}

The ability to identify the primary visual cortex and its monocular and binocular zones in any one of three different preparations of normal histological material (Nissl, myelin, or acetylcholinesterase) will prove useful for future studies of cortical plasticity and function in the mouse.

As initially reported by Dräger (1974), the position and extent of the mouse visual cortex, and its binocular component, are most clearly visible in experiments using transneuronal labeling of geniculocortical projections to the two hemispheres. The pattern of labeling contralateral to the injected eye reveals that the geniculocortical projection reaches the posterior margin of the hemisphere (consistent with the map of Wagor et al., 1980), as it does in the rat (Ribak and Peters, 1975; Zilles et al., 1984). In the most posterior coronal sections, the visual cortex thus extends into the medial aspect of the hemisphere, which must be part of the monocular zone of the visual cortex, because transneuronal labeling of the ipsilateral hemisphere was confined to more lateral regions. We believe that the labeling in the ventromedial part of the hemisphere is not an artifact attributable to contamination from high-intensity labeling of the tectum, as Dräger (1974) suggested from her transneuronal experiments using tritiated compounds, because the most anterior sections in our material are not labeled despite heavy labeling of the contiguous tectum and pretectum (Fig. 2). The transneuronal experiments also demonstrate that in the binocular zone of the visual cortex, geniculocortical connections serving the two eyes are completely superimposed. Labeling was homogeneous, with no suggestion of segregation of the inputs serving the two eyes in either coronal or 
flattened tangential sections, consistent with previous work (mouse: Dräger, 1974, 1978; rat: Ribak and Peters, 1975; Zilles et al., 1984) and with other approaches used in the present paper, including intrinsic signal imaging and reconstruction of single afferent arbors.

The most striking feature of the population of reconstructed geniculocortical arbors is the variety of morphological types. Arbors differed over a fivefold range in size and numbers of branch points and differed greatly in the laminar distribution of collateral branches, even when originating from a single LGN injection site (Fig. 8, see axons of $\mathrm{mo2}$ ). This is likely to reflect the labeling of heterogeneous relay cell types in the LGN (Fukuda, 1973).

\section{Effect of monocular deprivation}

The normal mouse visual cortex is dominated by the contralateral eye even in its binocular zone (Dräger, 1978; Dräger and Olsen, 1980; Gordon and Stryker, 1996) (Fig. 13, A, open symbols, B). As in other species, monocular visual deprivation has a powerful effect during a critical period in early life. However, the pronounced initial bias toward the contralateral eye profoundly influences the outcome of deprivation. The deprived eye loses nearly all of its influence on the responses of cells recorded in the ipsilateral hemisphere, and although the other hemisphere comes to be dominated by the open (ipsilateral) eye, the deprived (contralateral) eye retains the ability to drive almost all of the cells. Optical imaging studies confirmed the initial contralateral bias in normal animals, as well as the pronounced difference between the two hemispheres after MD. The optical responses of the deprived eye nearly disappeared in the ipsilateral hemisphere. Ipsilateral responses to the nondeprived eye became substantially greater than normal and even greater than those of the contralateral deprived eye (Fig. 12). The greater effects on ipsilateral eye responses are consistent with the operation of competitive mechanisms starting from the biased initial conditions. Brief MD of $4 \mathrm{~d}$ in duration has its maximal effect on single-unit responses at approximately P26 (Gordon and Stryker, 1996) and almost no effect after P40 (Fagiolini et al., 1998), but as in the cat (Daw et al., 1992), some degree of residual plasticity persists after the peak of the critical period and can be revealed by more prolonged periods of MD (Fig. 13A,E).

Anatomically, transneuronal labeling of the input to the cortex confirmed a reliable and strong effect only on the ipsilateral pathways and only after prolonged deprivation beyond the peak of the critical period. Higher-resolution anatomical studies involving the reconstruction and measurement of individual arbors were limited for technical reasons to the contralateral eye's pathways. Nevertheless, the axonal reconstructions revealed the plasticity of this initially dominant pathway both during and after the peak of the critical period. The major effect observed for earlier deprivations was a premature expansion of the open eye's arbors rather than a retraction of the deprived eye's arbors, which remained similar to normal. After more prolonged deprivations, the already large arbors serving the open eye appear to have reached a ceiling size and expanded only slightly. More prolonged deprivations, during a period when normal arbors were increasing in size, prevented further growth of the deprived eye arbors, leaving them significantly smaller than the open eye's arbors.

In the cat, the physiological effects of MD are fully expressed after 1-2 d of deprivation, and the morphological effects take four to five times as long; the retraction of the contralateral deprived eye's inputs becomes apparent after $4 \mathrm{~d}$ and is fully expressed after $7 \mathrm{~d}$ of MD, whereas expansion of the open eye's arbors takes longer (Antonini and Stryker, 1996). Therefore, a deprivation in the mouse of $20 \mathrm{~d}$ (or five times the duration of the effective brief MD) was deemed sufficient to reveal the retraction of the contralateral deprived eye's inputs if it had taken place.

The magnitude of the differences between deprived and nondeprived arbors in the mouse was nearly as great as that which we have measured earlier in the cat (mean total length changed 2 times in cat vs 1.7 times in mouse; number of branch points changed 2.8 times in cat vs 1.68 times in mouse; Antonini and Stryker, 1996). These differences were highly significant in the cat, in which the entire range of the arbor sizes in each experimental condition spanned a factor of $\sim 2$. In the mouse, the range of arbor sizes spans a factor of $>5$, reducing the statistical significance of some of the changes that take place with development or deprivation.

\section{Mechanisms of plasticity}

Comparison of the effects of monocular and binocular deprivation on single-unit responses revealed that the plasticity of ocular dominance in the mouse results from a competitive interaction (Gordon and Stryker, 1996). The present findings of differences between the binocular and monocular segments in the effects of monocular deprivation as measured using transneuronal transport or optical imaging confirm this view.

Single-unit responses or visual cortical responses measured with optical imaging are the product of the integration of thalamocortical inputs and intrinsic corticocortical circuits. Plastic changes as a result of MD may take place at both of these stages (Valverde, 1967, 1968). Indeed, there is evidence that local inhibitory cortical circuits in the mouse drive the plasticity after MD (Hensch et al., 1998b). The evidence that physiological measurements showed greater effects of MD than the anatomical approaches (transneuronal labeling and single arbors reconstruction) probably reflects the fact that the latter examine only the input to the cortex, whereas the physiological measurements also reflect changes in intracortical circuits.

\section{Comparison with the cat}

It may be useful to summarize similarities and differences between plasticity in the visual cortex of cat and mouse. In both species, the major effect of MD is competitive, as discussed above. In both species, physiological changes are relatively fast, taking place over no more than a few days, whereas anatomical effects on thalamocortical inputs take longer. Both species have a critical period that peaks around the end of the fourth week of postnatal life (after gestation periods of quite different duration), followed by several weeks (in the mouse) or months (in the cat) of greatly reduced plasticity. Thalamocortical input arbors in both species continue to expand well past the end of the critical period during normal development.

The mouse differs from the cat in that the contralateral pathways are very much more dominant in the mouse, both anatomically and physiologically (Dräger, 1975, 1978; Dräger and Olsen, 1980; Gordon and Stryker, 1996), although the contralateral projection in the cat is physiologically quite dominant before the beginning of the critical period (Crair et al., 1998). The mouse also differs in that the major effect of deprivation on the contralateral projection is an arrest of growth rather than a prompt retraction of branches, as in seen in the cat (Antonini and Stryker, 1996). However, transneuronal labeling suggests that also the ipsilateral projection probably does retract in the mouse. The 
mouse geniculocortical arbors were much more heterogeneous in size, complexity, and laminar targets than in the cat. This difference may reflect in part a difference in the diversity of cell types at the injection sites studied. If we were able to label a sample of the entire geniculocortical projection in the cat, we might have found similar heterogeneity.

The mouse also differs in that it appears to lack the orientation and ocular dominance columns that are such a prominent feature of the organization of the cat's visual cortex. Fluctuations in the anatomical labeling and in intrinsic signal optical responses within the monocular or binocular zones were not consistent among animals and were no greater than the fluctuations observed within a single ocular dominance column in the cat studied with the same techniques. The lack of columns in mouse visual cortex should not, however, be regarded as definitive, because it remains possible that a finer scale columnar organization, or perhaps a columnar organization of some other feature, will one day be discovered in the mouse. In addition, it is possible to regard the binocular and monocular zones of the mouse visual cortex as structures analogous to a single contralateral eye ocular dominance column in the cat plus a border region within which inputs serving the two eyes are mixed. This view would be appropriate in relation to the sizes of individual axonal arbors in the two species, although it is inappropriate in relation to topographic organization.

The experimental approaches that have been used to study the visual cortex of the cat-single-unit recording, optical imaging, transneuronal labeling, and axon arbor reconstruction (Hubel and Wiesel, 1962; Shatz and Stryker, 1978; Humphrey et al., 1985; Antonini et al., 1998) — are shown here to provide useful information about development and plasticity in the mouse. The similarities between cat and mouse are profound. It seems likely that the differences between the two species in outcome of monocular deprivation may reflect the operation of mechanisms that are identical, beginning from the different initial conditions that are present at the start of the critical period.

\section{REFERENCES}

Antonini A, Stryker MP (1996) Plasticity of geniculocortical afferents following brief or prolonged monocular occlusion the cat. J Comp Neurol 369:64-82.

Antonini A, Stryker MP (1998) Effect of sensory disuse on geniculate afferents to cat visual cortex. Vis Neurosci 15:401-409.

Antonini A, Gillespie DC, Crair MC, Stryker MP (1998) Morphology of single geniculocortical afferents and functional recovery of the visual cortex after reverse monocular deprivation in the kitten. J Neurosci 18:9896-9909.

Blakemore C, Vital-Durand F, Garey LJ (1981) Recovery from monocular deprivation in the monkey. I. Reversal of physiological effects in the visual cortex. Proc R Soc Lond B Biol Sci 213:399-423.

Bonhoeffer T, Grinvald A (1993) Optical imaging of the functional architecture in cat visual cortex: the layout of direction and orientation domains. Adv Exp Med Biol 333:57-69.

Caviness Jr VS (1975) Architectonic map of neocortex of the normal mouse. J Comp Neurol 164:247-263.

Caviness VS, Frost DO (1980) Radial organization of thalamic projections to the neocortex in the mouse. J Comp Neurol 194:369-393.

Crair MC, Ruthazer ES, Gillespie DC, Stryker MP (1997) Relationship between the ocular dominance and orientation maps in visual cortex of monocularly deprived cats. Neuron 19:307-318.

Crair MC, Gillespie DC, Stryker MP (1998) The role of visual experience in the development of columns in cat visual cortex. Science 279:566-570.

Daw NW, Fox K, Sato H, Czepita D (1992) Critical period for monocular deprivation in the cat visual cortex. J Neurophysiol 67:197-202.

Dräger UC (1974) Autoradiography of tritiated proline and fucose transported transneuronally from the eye to the visual cortex in pigmented and albino mice. Brain Res 82:284-292.

Dräger UC (1975) Receptive fields of single cells and topography in mouse visual cortex. J Comp Neurol 160:269-290.

Dräger UC (1978) Observations on monocular deprivation in mice. J Neurophysiol 41:28-42.

Dräger UC, Olsen JF (1980) Origin of crossed and uncrossed retinal projections in the pigmented and albino mice. J Comp Neurol 191:383-412.

Fagiolini M, Pizzorusso T, Berardi N, Domenici L, Maffei L (1994) Functional postnatal development of the rat primary visual cortex and the role of visual experience: dark rearing and monocular lid suture. Vision Res 34:709-720.

Fagiolini M, Antonini A, Stryker MP (1997) Shape and arrangement of geniculocortical afferents in the mouse. Soc Neurosci Abstr 23:83.

Fagiolini M, Mataga N, Baekkeskov S, Kosh SF, Hensch TK (1998) Disruption of the GAD65 gene preserves plasticity beyond the critical period in the primary visual cortex. Soc Neurosci Abstr 24:419.5.

Franklin KBJ, Paxinos G (1997) The mouse brain in stereotaxic coordinates. San Diego: Academic.

Fukuda Y (1973) Differentiation of principal cells of the rat lateral geniculate body into two groups: fast and slow cells. Exp Brain Res 17:242-260.

Gallyas F (1979) Silver staining of myelin by means of physical development. Neurol Res 1:203-209.

Goodman CS, Shatz CJ (1993) Developmental mechanisms that generate precise patterns of neuronal connectivity. Cell [Suppl] 72:77-98.

Gordon JA, Stryker MP (1996) Experience-dependent plasticity of binocular responses in the primary visual cortex of the mouse. J Neurosci 16:3274-3286.

Guillery RW (1972) Binocular competition in the control of geniculate cell growth. J Comp Neurol 144:117-129.

Guillery RW (1973) The effect of lid suture upon the growth of cells in the dorsal lateral geniculate nucleus of kittens. J Comp Neurol 148:417-422.

Guillery RW, Stelzner DJ (1970) The differential effects of unilateral lid closure upon the monocular and binocular segments of the dorsal lateral geniculate nucleus in the cat. J Comp Neurol 139:413-421.

Hata Y, Stryker MP (1994) Control of thalamocortical afferent rearrangement by postsynaptic activity in developing visual cortex. Science 265:1732-1735.

Hedreen JC, Bacon SJ, Price DL (1985) A modified histochemical technique to visualize acetylcholinesterase-containing axons. J Histochem Cytochem 33:134-140.

Hensch TK, Gordon JA, Brandon EP, McKnight GS, Idzerda RL, Stryker MP (1998a) Comparison of plasticity in vivo and in vitro in the developing visual cortex of normal and protein kinase A RIbetadeficient mice. J Neurosci 18:2108-2117.

Hensch TK, Fagiolini M, Mataga N, Stryker MP, Baekkeskov S, Kash SF (1998b) Local GABA circuit control of experience-dependent plasticity in developing visual cortex. Science 282:1504-1507.

Hubel DH, Wiesel TN (1962) Receptive fields, binocular interactions and functional architecture in the cat's visual cortex. J Physiol (Lond) 160:106-154.

Hubel DH, Wiesel TN (1965) Binocular interaction in striate cortex of kittens reared with artificial squint. J Neurophysiol 28:1041-1059.

Hubel DH, Wiesel TN (1970) The period of susceptibility to the physiological effects of unilateral eye closure in kittens. J Physiol (Lond) 206:419-436.

Hubel DH, Wiesel TN, LeVay S (1977) Plasticity of ocular dominance columns in monkey striate cortex. Philos Trans R Soc Lond B Biol Sci 278:131-163.

Humphrey AL, Sur M, Ulrich DJ, Sherman SM (1985) Projection patterns of individual $\mathrm{X}$ - and $\mathrm{Y}$-cell axons from the lateral geniculate nucleus to cortical area 17 in the cat. J Comp Neurol 233:159-189.

Itaya SK, van Hoesen GW (1982) WGA-HRP as a transneuronal marker in the visual pathways of monkey and rat. Brain Res 236:199-204.

LeVay S, Wiesel TN, Hubel DH (1980) The development of ocular dominance columns in normal and visually deprived monkeys. J Comp Neurol 191:1-51.

Mesulam MM (1978) Tetramethyl benzidine for horseradish peroxidase neurohistochemistry: a non-carcinogenic blue reaction product with superior sensitivity for visualizing neural afferents and efferents. J Histochem Cytochem 6:106-117. 
Movshon JA (1976) Reversal of the physiological effects of monocular deprivation in the kitten's visual cortex. J Physiol (Lond) 261:125-174.

Olavarria J, Montero VM (1989) Organization of visual cortex in the mouse revealed by correlating callosal and striate-extrastriate connections. Vis Neurosci 3:59-69.

Passera A, Fulks S, Schneider GE, Ayres S, Jhaveri S, Erzurumlu RS (1988) The MIT "Neurotrace" system for computer-aided microscopy. Soc Neurosci Abstr 14:550.

Reese BE (1988) "Hidden lamination" in the dorsal lateral geniculate nucleus: the functional organization of this thalamic region in the cat. Brain Res Rev 13:119-137.

Reese BE, Cowey A (1983) Projection lines and the ipsilateral retinogeniculate pathway in the hooded rat. Neuroscience 10:1233-1247.

Reese BE, Jeffery G (1983) Crossed and uncrossed visual topography in the dorsal geniculate nucleus in the pigmented rat. J Neurophysiol 49:877-885.

Reiter HO, Stryker MP (1988) Neural plasticity without postsynaptic action potentials: less-active inputs become dominant when kitten visual cortical cells are pharmacologically inhibited. Proc Natl Acad Sci USA 85:3623-3627.

Ribak CE, Peters A (1975) Autoradiographic study of the projections from the lateral geniculate body of the rat. Brain Res 92:341-368.

Shatz CJ, Stryker MP (1978) Ocular dominance in layer IV of the cat's visual cortex and the effects of monocular deprivation. J Physiol (Lond) 281:267-283
Simmons PA, Lemmon V, Pearlman AL (1982) Afferent and efferent connections of the striate and extrastriate visual cortex of the normal and reeler mouse. J Comp Neurol 211:295-308.

Stryker MP, Harris WA (1986) Binocular impulse blockade prevents the formation of ocular dominance columns in cat visual cortex. J Neurosci 6:2117-2133.

Valverde F (1967) Apical dendrites spines of the visual cortex and light deprivation in the mouse. Exp Brain Res 3:337-352.

Valverde F (1968) Structural changes in the area striata of the mouse after enucleation. Exp Brain Res 5:274-292.

van Sluyters RC (1978) Reversal of the physiological effects of brief periods of monocular deprivation in the kitten. J Physiol (Lond) 284:1-17.

Wagor E, Mangini NJ, Pearlman AL (1980) Retinotopic organization of striate and extrastriate visual cortex in the mouse. J Comp Neurol 193:187-202.

Wiesel TN, Hubel DH (1963) Single cell responses in striate cortex of kittens deprived of vision in one eye. J Neurophysiol 28:1029-1040.

Wiesel TN, Hubel DH (1965a) Comparison of the effects of unilateral and bilateral eye closure on cortical unit responses in kittens. J Neurophysiol 28:1029-1040.

Wiesel TN, Hubel DH (1965b) Extent of recovery from the effects of visual deprivation kittens. J Neurophysiol 28:1060-1072.

Zilles K, Wree A, Schleicher A, Divac I (1984) The monocular and binocular subfields of the rat's primary visual cortex: a quantitative morphological approach. J Comp Neurol 226:391-402. 